\title{
Vibration Control by Means of Piezoelectric Actuators Shunted with LR Impedances: Performance and Robustness Analysis
}

\author{
M. Berardengo, ${ }^{1}$ A. Cigada, ${ }^{1}$ S. Manzoni, ${ }^{1}$ and M. Vanali ${ }^{2}$ \\ ${ }^{1}$ Department of Mechanical Engineering, Politecnico di Milano, Via La Masa 34, 20156 Milan, Italy \\ ${ }^{2}$ Department of Industrial Engineering, Università degli Studi di Parma, Parco Area delle Scienze 181/A, 43124 Parma, Italy \\ Correspondence should be addressed to S. Manzoni; stefano.manzoni@polimi.it
}

Received 29 October 2014; Revised 8 April 2015; Accepted 14 April 2015

Academic Editor: Lei Zuo

Copyright (c) 2015 M. Berardengo et al. This is an open access article distributed under the Creative Commons Attribution License, which permits unrestricted use, distribution, and reproduction in any medium, provided the original work is properly cited.

\begin{abstract}
This paper deals with passive monomodal vibration control by shunting piezoelectric actuators to electric impedances constituting the series of a resistance and an inductance. Although this kind of vibration attenuation strategy has long been employed, there are still unsolved problems; particularly, this kind of control does suffer from issues relative to robustness because the features of the electric impedance cannot be adapted to changes of the system. This work investigates different algorithms that can be employed to optimise the values of the electric components of the shunt impedance. Some of these algorithms derive from the theory of the tuned mass dampers. First a performance analysis is provided, comparing the attenuation achievable with these algorithms. Then, an analysis and comparison of the same algorithms in terms of robustness are carried out. The approach adopted herein allows identifying the algorithm capable of providing the highest degree of robustness and explains the solutions that can be employed to resolve some of the issues concerning the practical implementation of this control technique. The analytical and numerical results presented in the paper have been validated experimentally by means of a proper test setup.
\end{abstract}

\section{Introduction}

Mitigation of vibrations in structures is a crucial issue in several fields, such as electronics, automotive, space, and manufacturing. It can lead to higher quality products, it improves durability by protecting components from fatigue and failure, it achieves reduced maintenance costs, and it improves comfort to people in terms of noise and vibration.

In this scenario, the control of light structures is of particular importance. Many industrial and engineering applications indeed rely on lightweight structures subject to a harsh dynamic environment. Usually these structures have low damping values: thus the vibration level induced can be very high. Furthermore, they are lightweight so that the actuators used for control purposes often introduce high load effects.

Due to these issues, many active and passive strategies have been developed to increase the damping of these structures. Among the passive strategies, up to 20 years ago, the most common were the introduction of high loss factor viscoelastic materials within the structure or connection to a mechanical vibration absorber. In 1991 Hagood and von Flotow [1] proposed a new method to passively increase the structural damping, by relying on piezoelectric materials shunted to a proper electrical network. Since this advance, a variety of passive and semipassive vibration reduction techniques based on piezoelectric materials have been proposed.

The capability of piezoelectric materials to convert mechanical energy into electrical and vice versa can indeed be employed in different ways: they can be used as sensors or actuators for active and passive control strategies. As regards the passive or semipassive control techniques, these materials allow either dissipating or reusing the electrical energy induced by the mechanical deflection, by means of a suitable electrical device. In the latter case, an electrical network has to be properly designed and shunted to a piezoactuator, in order to generate an action opposite to the motion of the hosting structure.

The simplest passive shunt-circuits for single mode control are the resistive $(R)$ and the resistive-inductive ( $L R$ or resonant shunt, in which a resistance and an inductance are 
connected in series), which are the electrical equivalents of a Lanchester damper and a Tuned Mass Damper (TMD), respectively $[2,3]$. Such network layouts are widely used for their effectiveness. In addition to these circuits, other types of shunt impedance have been developed; among others, $\mathrm{Wu}$ [4] has suggested using a resistance and an inductance in parallel, and Park and Inman [5] studied together both series and parallel $L R$ circuits.

These passive techniques based on shunted piezoelectric actuators are particularly appealing to the scope of suppressing vibration: they require no power to be effective, which even allows coupling them to energy harvesting systems. Moreover, they cause little additional weight: a strategic issue for most light structures (e.g., space structures). Furthermore, these methods do not require either digital or analog expensive control systems and feedback sensors; they are always stable and easy to implement. On the other hand, these approaches are less flexible than active control strategies: therefore they must be carefully designed and optimised for each specific application. It is also noticed that $R$ and $L R$ impedances can be coupled to a negative capacitance [68 ] in order to increase their attenuation performances. Such an approach allows increasing the vibration attenuation provided by the shunt impedance but poses some issues related to the stability of the electromechanical system because a circuit based on operational amplifiers is used to build the negative impedance. Therefore, the shunt impedance becomes semiactive.

Several strategies have been suggested to optimize the impedance parameters so to achieve the best performances from single mode controllers. Hagood and von Flotow [1] proposed tuning strategies based on transfer function criteria and on pole placement, to tune the numerical values of the $R L$ shunt impedance in undamped structures. Both these tuning methods are based on the classic TMD theory, given that the $R L$ circuit is the electrical equivalent of the TMD. In the first method abovementioned, that is, transfer function criterion, the inductance value is found by imposing some constraints on the transfer function shape of the coupled system. Instead, in the latter pole placement technique, the resistance and inductance values are set so that the complex poles of the shunted piezoelectric system reach the leftmost excursion in the s-plane. Høgsberg and Krenk developed a balanced calibration method for series and parallel $R L$ circuits, based on pole placement [9]. In this case, the $R$ and $L$ values are chosen in order to guarantee equal modal damping of the two modes of the electromechanical structure and thus good separation of complex poles. This option guarantees a good compromise between high damping and performance in terms of response reduction with limited damping. Successful empirical methods to tune the $R L$ impedance [10] have also been proposed in order to optimize the performance of the system, only with knowing the geometric, mechanical, and electrical characteristics of the hosting structure and actuator.

In addition to the impedance optimization, several studies have investigated the influence of the geometry and placement of the piezoelectric actuator on the performances of the controller. A number of studies to optimize geometry and position of the piezo-actuator have been carried out on the basis of finite element model analysis [11-14]. Instead Ducarne et al. proposed a method to optimise the geometry and the placement of the piezo-actuator, in order to increase the damping efficiency by maximizing the Modal ElectroMechanical Coupling Factor (MEMCF) [15]. Thomas et al. also proposed closed formulas to evaluate the performance of the controller as function of the MEMCF [16].

For several reasons the performances of these passive control strategies are lower than the ones employing active control, even though they are optimized so to achieve the best vibration attenuation: firstly the power involved in the control is lower than in the case of active control, secondly the absence of a feedback control linked to an error signal does not allow improving control performance during actuation. Consequently, these strategies are much more conditioned by the uncertainties and sensitive to the changes of the parameters involved, thus leading to poor results when the strategies are not well tuned to the specific application. Therefore, in most cases it is not possible to successfully apply the methods available in literature in order to choose the right values of the impedance parameters. In most of the cases the practical applications require empirical tuning or adjustment of the theoretical optimal values. This is in agreement with the results obtained by Thomas et al. in their experimental tests [16]. In fact, they had to adjust the theoretical values calculated with their optimization method in order to achieve the highest attenuation values. The uncertainty which affects the mechanical and electrical parameters of both the structure and the piezoelectric actuators is indeed extremely high in the practical application. Furthermore, mistuning can occur even when starting from a perfectly tuned condition: for instance, if the environmental temperature changes, the eigenfrequency of the system to control will shift and a mistuning will thus occur. Therefore, the chances of having to work in mistuned conditions are very high in practical cases and this causes worse vibration attenuation performance.

Some techniques based on adaptive circuits have been proposed to overcome the limitations due to uncertainty on mechanical and electrical quantities leading to mistuning. As regarding the single mode control, Hollkamp and Starchville developed a self-tuning $R L$ circuit able to follow any change in frequency of the mode to control [17]. This technique is based on a synthetic circuit (which provides both the inductance and the resistance of the circuit) constituted by two operational amplifiers and a motorized potentiometer. A change of the input voltage to the motorized potentiometer results in a change of the electrical resonance, so that the control system can follow the mechanical resonance change, allowing the correct tuning of the impedance. Nevertheless, this circuit contains active components needing a power supply: thus this strategy cannot be considered passive. Furthermore, the only uncertainty taken into consideration in the abovementioned referenced work is the one relative to the frequency of the controlled mode, while the uncertainty relative to the shunt parameters and to the electrical quantities of the piezoactuator is not taken into account. Other recent works by Zhou et al. investigated methods to limit the problem of mistuning, by binding more than one piezo-actuator to 
the vibrating structure [18] and by employing nonlinear elements when the disturbance is harmonic [19].

Although the passive control strategies by means of piezoelectric actuators have been widely studied in the last twenty years, there is still much need for improvement, because of some criticalities. The most relevant ones are summarized below and will each be discussed in detail further in this section:

(1) Most of the methods to tune the shunt impedance, available in literature, require the estimation of the natural frequency of the electromechanical system in open and short circuit conditions or the estimation of the Electro-Mechanical Coupling Factor (EMCF).

(2) In most cases, the impedance optimization algorithms provide numerical values of the parameters, which are nonetheless unfeasible in practice. As for $R L$ tuning, very large inductors are necessary for the more commonplace mechanical frequencies; moreover the resistance values are often so small that the sole resistance of the cables and of the piezo-actuator [20] together results to be higher than the value of the optimal shunt resistance itself. Therefore, it is necessary to implement synthetic circuits by means of operational amplifiers, in order to overcome the limitation due to the high value of the inductance $L$. This solution nonetheless leads to the problem that this synthetic circuit requires power supply. Moreover, the problem of the low value of the resistance has seldom been studied in literature.

(3) Although it is well known [21-23] that the performance of the control strategy varies significantly in case of uncertainty on the mechanical and electrical parameters of both structure and actuator, a robustness analysis has not yet been carried out in any of the works available in literature. Furthermore, the behaviour of the optimisation methods, in case of mistuning, has never been analysed in terms of attenuation performances.

The aim of this paper is thus to resolve some of these shortcomings.

Relative to the issue in point 1 of the list presented above, the problem should be broken down into different considerations. Firstly, when a numerical estimate of the EMCF value is needed, approximated closed formulas may be used when available [15] or else it is possible to measure the natural frequency of the short and open circuit. In this latter case the piezo-actuator has to be chosen and bonded a priori and only subsequently the impedance can be tuned. Therefore, the optimization procedure is carried out in two different steps: the optimization of the actuator placement and the optimisation of the impedance parameters. Though some optimization methods are available for the placement and the geometry of the actuator [11-15], this procedure precludes the possibility to perform a more general analysis taking into account at the same time the shunt-impedance parameters, the geometric parameters, and the position of the actuator. But such an analysis can be of great importance for a number of reasons: a specific desired performance may be achieved by different configurations, not necessarily the optimum one. Moreover, sometimes a solution comparable to the optimal one in terms of vibration reduction can also be achieved with an electromechanical configuration different from the optimal one in terms of geometry and position of the actuator, by properly tuning the impedance parameters. This solution can be hardly achieved if the optimization is carried out in two separate steps. A comprehensive analysis in which every parameter is optimized at the same time would also allow to estimate the performance of the controller a priori and therefore to highlight whether such a kind of control strategy can be effective enough or not.

The second point in the list of criticalities abovementioned concerns the values of $R$ and $L$ deriving from the optimization methods: the problem is that their values often result to be too small and too large, respectively, in order to be obtained by physical passive components. This leads to the need of implementing the impedance through operational amplifiers, in turn requiring power supply, even though the power necessary is actually very low. The comprehensive approach proposed above could clarify if the values of $R$ and $L$ can be changed in order to become feasible, by changing other system parameters (e.g., geometric, mechanical, and electrical parameters, position of the actuator) maintaining the same performance of the controller.

By combining this general analysis with an analysis of robustness to mechanical and electrical uncertainties, proposed in point number 3 of the list of criticalities, a clearer and complete insight on the problem under analysis can be achieved.

This paper proposes an analytical treatment that enables the user to investigate all these aspects. A comprehensive approach, as discussed above, has been developed: it aims at obviating to the aforementioned criticalities by sustaining the tuning algorithm by means of a performance and robustness analysis.

The model employed to describe the dynamic behaviour of the coupled electromechanical system plays an important role in the development of this procedure. It must provide clear formulations which allow performing a global analysis highlighting simultaneously the influence of the position of the piezo-actuator, of its geometry, and of the shunt impedance parameters. Then, all the analyses underlined before can be carried out.

This model chosen takes advantage of the one proposed by Moheimani and Fleming [3] and has several benefits. Firstly the control action performed by the shunted electrical network is seen as a feedback loop: this allows applying the classic control theory to the electromechanical system. Moreover, this model is able to describe at the same time the behaviour of both the elastic structure and the piezoelectric actuator, which in turn are coupled with the shunted impedance. This kind of modelling takes into account both the electromechanical structure (piezoactuator + structure) and the shunt impedance. Also, this model can describe, with a single mathematical description, both 1-dimensional (e.g., beams) and 2-dimensional (e.g., plates) structures. 


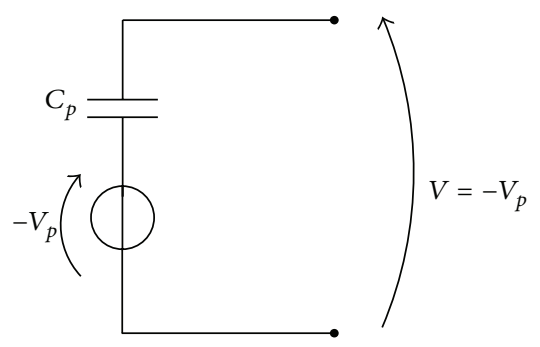

(a)

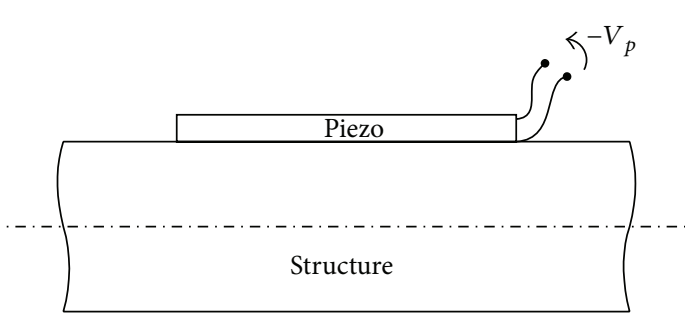

(b)

FIGURE 1: Electric equivalent of a piezoelectric actuator (a) bonded to an elastic structure (b).

By the use of this analytical model, this paper demonstrates that there is one specific parameter which affects the control performances and the effectiveness of the control strategy. Such a parameter depends only on the mechanical, geometrical, and electrical characteristics of the structure and of the actuator. This parameter, together with the shunt impedance parameters, can be then modified and properly tuned in order to achieve the target performances. As explained above, the simultaneous tuning of these parameters can be advantageous. Furthermore, this approach brings to light the ineffectiveness of the control techniques based on shunted piezoelectric actuators in the cases where the natural frequencies and the damping of the mode to be controlled exceed given values.

In this scenario, three different methodologies to tune the impedance parameters have been developed, all relying on transfer function considerations. Analytic closed formulas to derive the optimal values of the resistance and inductance of the $R L$ shunt circuit for damped light structures were then derived. Although all of these strategies prove to be very effective when there are no uncertainties on the parameters, a robustness analysis shows that one of these three tuning algorithms is more robust than the others to uncertainties on electrical and mechanical parameters.

All the results have been experimentally validated. Since the approach developed in this paper results is valid for both beams and plates, the authors have decided to build a test setup, with an aluminium plate and a piezo patch bonded close to its centre, which provides a more complex case-study than those commonly treated in literature (i.e., often the 1dimensional case is preferred).

This paper is structured as follows. The general electromechanical model for an elastic structure with piezoelectric elements coupled to an electric circuit is described in Section 2. The three different tuning methodologies based on transfer function considerations are presented in Section 3, and analytic formulas to tune $R$ and $L$ are derived. Section 4 illustrates the performance analysis of the mentioned optimisation methods and explains the effect of the electric and mechanical characteristics of the structure and of the piezoactuator on the $R$ and $L$ values. The robustness analysis of the optimization methods is presented in Section 5. Finally, Section 6 illustrates and explains the experimental tests carried out on a plate, and a simplified formulation for the most robust tuning procedure is proposed in Section 7.

\section{Electromechanical Model}

This section treats the analytical modelling of the whole electromechanical structure constituted by the elastic structure, the piezo-actuator, and the shunting impedance. Though some of the issues treated in this section are already known and discussed in literature $[3,24,25]$, the authors have decided to provide a concise recapitulation of them, for sake of clarity; such an abridgement is moreover meant to make the paper more readily accessible and makes for a better understanding of the improvements contributing to such a model by this paper. This section is subdivided into four parts: the first part describes the electric model of the piezoelectric actuator is described; part two highlights the feedback nature of the controlled system; and the third part provides the dynamic model of the coupled system, and analytical formulations are derived for cases that have yet to be analysed in literature. Finally, this model is used to achieve a new formulation of the frequency response function of the controlled structure, in the fourth subsection.

2.1. Electric Equivalent Scheme of a Piezoelectric Actuator. Piezoelectric materials are materials such that an applied stress is capable of generating a charge on the surfaces of the piezoelectric element, and an applied voltage generates a strain. Thanks to the latter working principle, the shape of the solid can be modified depending on the charge induced on the surfaces of the piezoelectric element. These two effects (called piezoelectric effects, direct and inverse, resp.) entail to employ these materials as both sensors and actuators, making them extremely interesting in applications for vibration control.

One of the models which can be used to describe the electrical behaviour of piezoelectric materials is a series of a capacitor $C_{p}$ and a strain-dependent voltage generator $-V_{p}$ [26], as shown in Figure 1.

Two piezoelectric patches are usually needed to control a light structure by means of piezoelectric materials: one acting as sensor and the other as actuator (Figure 2). The sensor output (i.e., the voltage $V$ across the piezoelectric patch electrodes) is equal to $-V_{p}$ (Figure 1) and it depends on the strain of the structure to which it is bonded. In turn, this voltage is the input to the controller which uses this signal as the reference for its control law, generating an output voltage 


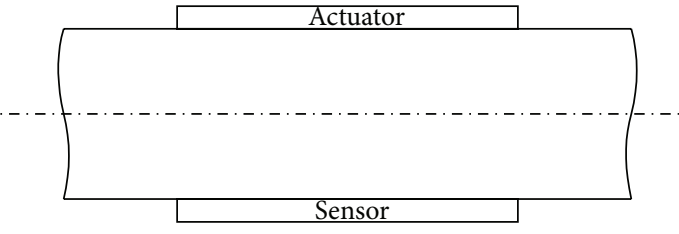

(a)

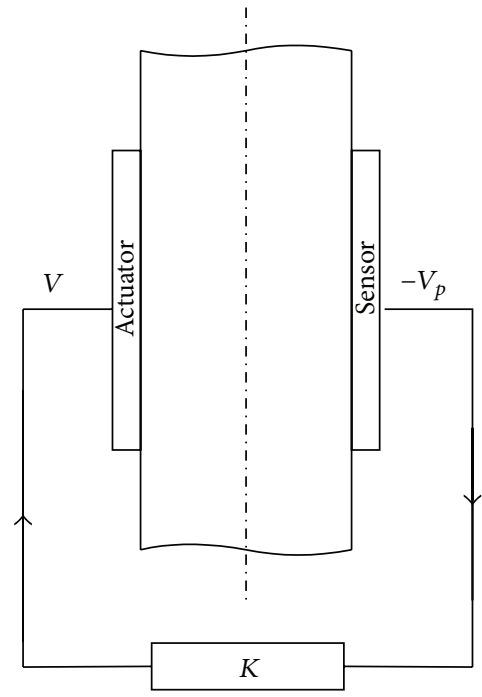

(b)

FIgURE 2: Scheme of an elastic structure controlled by means of coupled piezoelectric sensor and actuator.

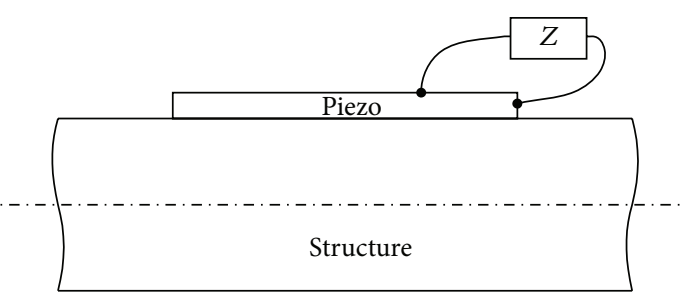

(a)



(b)

FIGURE 3: Scheme of a structure controlled by a shunted piezo-actuator (a) and its electrical equivalent (b).

$V$, applied to the piezo-patch acting as actuator. This piezoactuator will thus change its shape, thus applying a control force to the structure.

The control mechanism described here can also be made by using a single piezo-patch, which behaves at the same time as sensor and actuator. In this case a single piezoelectric element is shunted to an impedance $Z$ [3] (Figure 3); the structure vibration will induce a voltage $V$ to the terminals of the actuator (equal to $-V_{p}$ when the circuit is open: see Figure 1). Since the impedance $Z$ is connected to the piezoelectric element, a current $i_{z}$ circulates and the voltage $V$ between the terminals of the impedance $Z$ will no longer be $-V_{p}$, but it will be modified by the presence of the impedance. This voltage becomes the input to the piezo-element and thus induces a change of the strain of the patch (i.e., a control force is imposed to the structure). Hence, the voltage $V$ and the control action to the structure depend on how the impedance used to shunt the actuator is built. Thus, its layout and the values of its parameters must be carefully chosen, according to the specific application, in order to maximize the control effect.
This kind of control scheme (Figure 3) is chosen to develop the single mode control strategies presented in this paper.

2.2. Feedback Representation of the Control by Means of a Shunted Piezoelectric Actuator. Taken here into consideration is a structure controlled by a piezoelectric actuator shunted by an impedance $Z$ and subject to the disturbance $W$ (Figure 4(a)) and its electrical equivalent, as shown in Figure 4(b). The disturbance $W$ induces a flexural motion of the elastic structure described by the variable $z$, which represents the transversal displacement. As explained in [27], if the impedance $Z$ is removed (open circuit), the voltage $V$ at the terminals of the actuator results equals $-V_{p}$ (Figure 1(b)) and is entirely induced by the strain generated by the disturbance $W$. In this case the voltage $-V_{p}$ can be related to the disturbance $W$ by the transfer function $G_{v w}$ :

$$
-V_{p}(s)=G_{v w} W(s) .
$$

Otherwise, in the case no disturbances act on the structure and the impedance $Z$ is replaced by a voltage source $V$; 


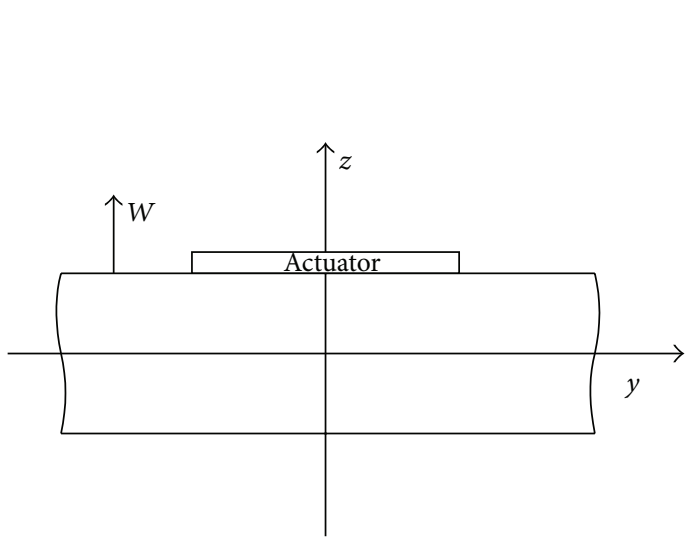

(a)

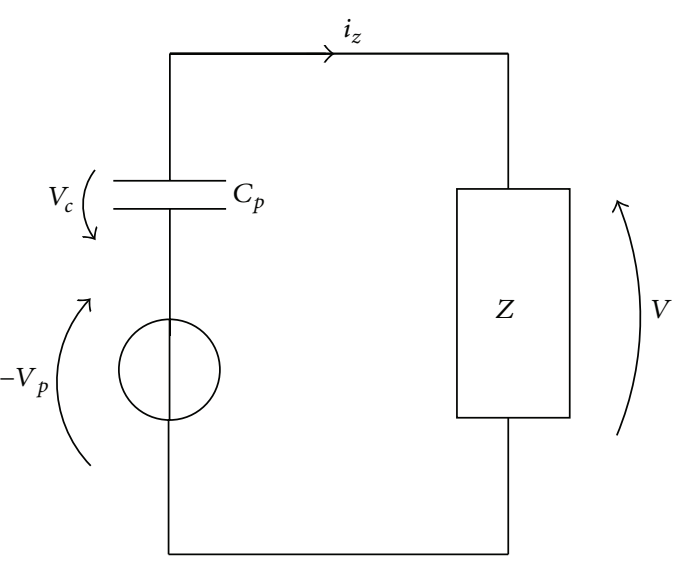

(b)

FIGURE 4: Structure controlled by a piezoelectric actuator shunted by the impedance $Z$ and subject to the disturbance $W$ (a) and its electrical equivalent (b).

then $-V_{p}$ depends only on the voltage $V$. Thus, the relation between $V$ and $-V_{p}$ can be expressed by the transfer function $G_{v v}$ :

$$
-V_{p}(s)=G_{v v} V(s) .
$$

When the disturbance $W$ acts on the structure and the voltage $V$ is applied to the terminals of the piezo-actuator, the voltage $-V_{p}$ can be expressed as the sum of the two contributions, because of the linearity of the system. By applying the superposition principle, it is then possible to write

$$
-V_{p}(s)=G_{v v} V(s)+G_{v w} W(s) .
$$

As for Figure 4(b), the following expressions are achieved by applying the Kirchhoff and the Ohm laws:

$$
\begin{aligned}
& V_{c}=-V_{p}-V, \\
& V=Z i_{z},
\end{aligned}
$$

where $V_{c}=i_{z} / C_{p} s$ in the Laplace domain.

Equations (3) and (4) allow representing the electromechanical structure, constituted by the elastic structure, the piezo-patch, and the impedance, as the feedback controlled system in Figure 5, where the controller $K$ in the Laplace domain is represented by the inner loop:

$$
K(s)=\frac{V}{-V_{p}}=\frac{s C_{p} Z}{1+s C_{p} Z} .
$$

Therefore, the impedance $Z$ can be used to produce a given voltage $V$ capable of reducing the effect induced by the disturbance $W$. The impedance used in this paper to develop a single mode control strategy is constituted by a resistance $R$ in series with an inductance $L$.

As mentioned above, different tuning methodologies will be defined in Section 3, in order to choose the optimal values of the impedance parameters (i.e., $R$ and $L$ ), relying on

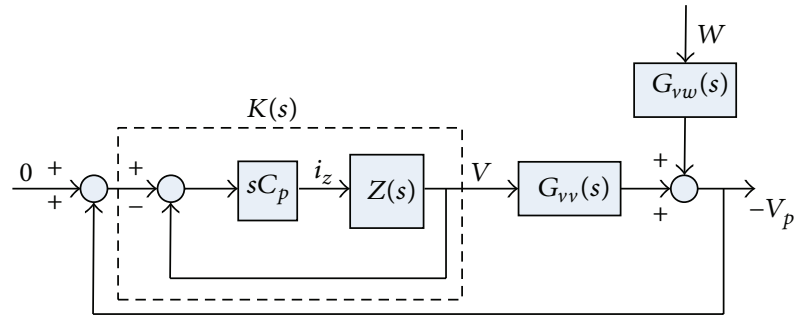

FIGURE 5: Feedback representation of the controlled structure.

transfer function considerations. The selected target transfer function shall allow describing the response of the elastic structure in terms of deflection to an external disturbance. Therefore, the expression of the closed loop transfer function $T_{z w}$ between the disturbance $W$ and the displacement $z$ of the structure is needed.

As for Figure 5, the closed loop transfer function $T_{v w}$ relating the voltage $-V_{p}$ with the disturbance $W$ can be defined as

$$
\begin{aligned}
-V_{p} & =G_{v w} W+G_{v v} V \\
& \Longrightarrow-V_{p}=G_{v w} W+G_{v v} K\left(-V_{p}\right) \\
& \Longrightarrow T_{v w}=\frac{-V_{p}}{W}=G_{v w} \frac{1}{1-K G_{v v}}
\end{aligned}
$$

Once the transfer function $G$ between the voltage $V$ and the displacement $z$ (i.e., $G(s)=z / V$ ) is defined, $T_{z w}$ can be expressed as

$$
T_{z w}=\frac{z}{W}=T_{v w} \frac{G}{G_{v v}}=G_{v w} \frac{1}{1-K G_{v v}} \cdot \frac{1}{G_{v v}} G .
$$

Thus, the analytic expressions of $G, G_{v v}$, and $G_{v w}$ must be known, in order to derive the expression of $T_{z w}$ and thus the dynamics of the coupled system (i.e., piezo-actuator + elastic structure) must be taken into account. 


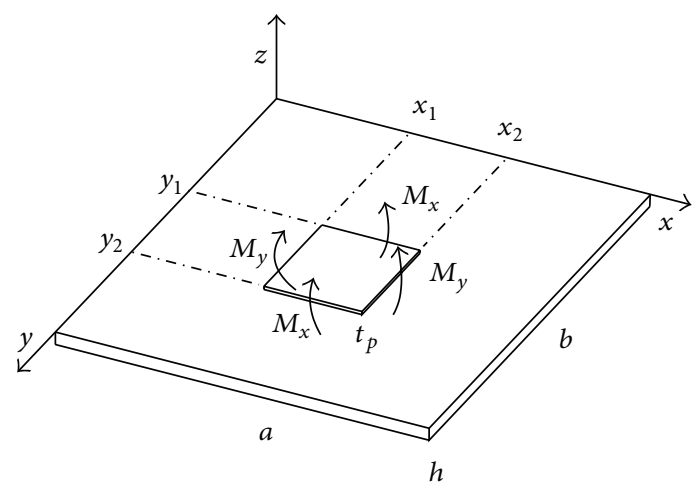

FIgURE 6: Plate structure with a piezo-element.

2.3. Dynamic Model of the Electromechanical System. The mathematical procedure, developed in this section, to derive the analytic relations between the electric and mechanical quantities involved in this control problem (i.e., $V,-V_{p}, z$, and $W$ ) is referred to a plate structure (Figure 6). This same approach can be used to derive these relationships for onedimensional cases (i.e., beam structures) as well $[24,25]$. The authors have decided to refer to the two-dimensional case but to report only the final results for the one-dimensional case, for sake of conciseness and because the experimental tests (Section 6) were performed on a plate.

The analytical formulation of the transfer functions $G$, $G_{v y}$, and $G_{v w}$ can be achieved by employing the expressions which relate the voltage induced at the terminals of the piezopatch in open circuit (i.e., $-V_{p}$ ) to its deformation and the equations of motion of the electromechanical system $[3,24]$.

The first of these equations (i.e., the relation between the voltage induced at the terminals of the piezo-patch in open circuit $-V_{p}$ and its deformation) can be derived considering that the voltage between the piezo-actuator terminals $-V_{p}$ in open circuit can be expressed as function of the charge $q$ induced on the surfaces of the patch:

$$
-V_{p}=\frac{q}{C_{p}}
$$

and the charge $q$ can be obtained as the surface integral of the electric displacement in $z$ direction, named $D_{3}$ :

$$
q=\int_{A_{p}} D_{3} d A_{p}
$$

where $A_{p}$ is the surface of the piezoelectric patch. From the equation describing the direct piezoelectric effect [3], the expression of the electric displacement $D_{3}$ can be derived as function of the stresses on the piezo-patch in $x$ and $y$ directions $\left(\sigma_{x}^{p}\right.$ and $\left.\sigma_{y}^{p}\right)$ and of the piezoelectric strain constants $d_{31}$ and $d_{32}$ :

$$
D_{3}=d_{31} \sigma_{x}^{p}+d_{32} \sigma_{y}^{p} .
$$

Assuming the piezoelectric material bears similar properties in $x$ and $y$ directions, the two piezoelectric constants can be considered equal: $d_{32}=d_{31}$. Furthermore, the stresses $\left(\sigma_{x}^{p}, \sigma_{y}^{p}\right)$ and strains $\left(\varepsilon_{P_{x}}, \varepsilon_{P_{y}}\right)$ in the piezoelectric patch in open circuit can be described by the following expressions:

$$
\begin{aligned}
\sigma_{x}^{p} & =\frac{E_{p}}{1-v_{p}^{2}}\left(\varepsilon_{P_{x}}+v_{p} \varepsilon_{P_{y}}\right), \\
\sigma_{y}^{p} & =\frac{E_{p}}{1-v_{p}^{2}}\left(\varepsilon_{P_{y}}+v_{p} \varepsilon_{P_{x}}\right), \\
\varepsilon_{P_{x}} & =\left(\frac{h}{2}+\frac{t_{p}}{2}\right) \frac{\partial^{2} w}{\partial x^{2}}, \\
\varepsilon_{P_{y}} & =\left(\frac{h}{2}+\frac{t_{p}}{2}\right) \frac{\partial^{2} w}{\partial y^{2}},
\end{aligned}
$$

where $h$ and $t_{p}$ are the thickness of the plate and of the piezoactuator, respectively, $E_{p}$ and $\nu_{p}$ are Young's and Poisson's moduli of the piezo-patch, and $w$ is the transverse deflection of the coupled structure.

By substituting (9), (10), and (11) in (8), the relation between the deformation of the piezoelectric patch and the voltage induced at its terminals is derived:

$$
-V_{p}=\frac{E_{p} d_{31}\left(h+t_{p}\right)}{2 C_{p}\left(1-v_{p}\right)} \int_{y_{1}}^{y_{2}} \int_{x_{1}}^{x_{2}}\left(\frac{\partial^{2} w}{\partial x^{2}}+\frac{\partial^{2} w}{\partial y^{2}}\right) d x d y,
$$

where $x_{1}, x_{2}, y_{1}$, and $y_{2}$ are the coordinates of the actuator extremities (Figure 6).

Now that the first equation has been derived (i.e., the relation between the voltage induced at the terminals of the piezo-patch in open circuit $-V_{p}$ and its deformation), the equations of motion of the plate are necessary to calculate the transfer functions $G, G_{v v}$, and $G_{v w}$.

As explained in the previous section, $G, G_{v v}$, and $G_{v w}$ represent the transfer functions related to the cases in which either the disturbance $W$ or the applied voltage $V$ is acting on the system. This means that the response of the elastic structure to the forces induced by the applied voltage $V$ and the disturbance $W$ separately must be known, in order to calculate these transfer functions.

The dynamic equation of a plate subject to a forcing term $W$ is represented by the following Partial Differential Equation (PDE) [28]:

$$
\rho h \frac{\partial^{2} w}{\partial t^{2}}+D \nabla^{4} w(x, y, t)=W,
$$

where $\rho$ is the density of the plate material and

$$
\begin{aligned}
\nabla^{4} w & =\frac{\partial^{4} w}{\partial x^{4}}+2 \frac{\partial^{4} w}{\partial x^{2} \partial y^{2}}+\frac{\partial^{4} w}{\partial y^{4}} \\
D & =\frac{E h^{3}}{12\left(1-v^{2}\right)}
\end{aligned}
$$

where $v$ and $E$ are Poisson's coefficient and Young's coefficient of the plate material, respectively. Equations (13) and (12) expressed in modal coordinates and represented in Laplace 




(a)

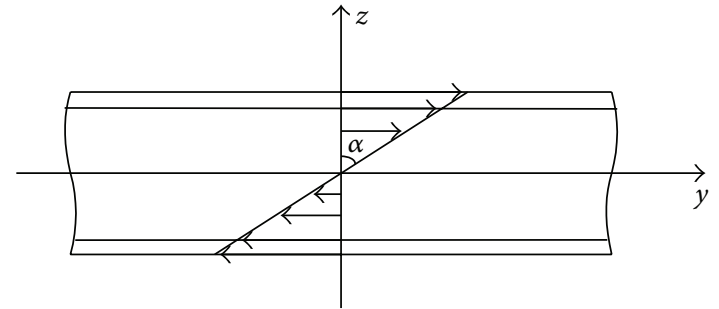

(b)

FIGURE 7: Two-dimensional strain distribution of a plate with two antisymmetric colocated piezoelectric elements: (a) $z-x$ plane and (b) $z-y$ plane.

domain allow calculating the transfer function $G_{v w}$ between the voltage $-V_{p}$ and the disturbance $W$ :

$$
G_{v w}=\frac{-V_{p}}{W}=\frac{E_{p} d_{31}\left(h+t_{p}\right)}{2 C_{p}\left(1-v_{p}\right)} \sum_{k=1}^{\infty} \frac{\Phi_{k}\left(x_{F}\right) \psi_{k}}{s^{2}+2 \xi_{k} \omega_{k} s+\omega_{k}^{2}},
$$

where $\omega_{k}$ is the $k$ th eigenfrequency of the plate and $\xi_{k}$ is the associated nondimensional damping ratio. $\Phi_{k}$ is the $k$ th eigenmode (normalised to unit modal mass) of the plate, $\Phi_{k}\left(x_{F}\right)$ represents the value of the $k$ th mode at the forcing point $x_{F}$, and $\psi_{k}$ for the two-dimensional case is

$$
\begin{aligned}
\psi_{k 2 D}= & \left.\int_{y_{1}}^{y_{2}} \frac{\partial \Phi_{k}(x, y)}{\partial x}\right|_{x=x_{2}} d y \\
& -\left.\int_{y_{1}}^{y_{2}} \frac{\partial \Phi_{k}(x, y)}{\partial x}\right|_{x=x_{1}} d y \\
& +\left.\int_{x_{1}}^{x_{2}} \frac{\partial \Phi_{k}(x, y)}{\partial y}\right|_{y=y_{2}} d x \\
& -\left.\int_{x_{1}}^{x_{2}} \frac{\partial \Phi_{k}(x, y)}{\partial y}\right|_{y=y_{1}} d x
\end{aligned}
$$

where $\psi_{k}$ is a term dependent on the curvature of the $k$ th mode and assumes different formulations for $1 \mathrm{D}$ or $2 \mathrm{D}$ cases; see Table 1 (the other parameters in Table 1 are explained in this section further on).

As for $G$ and $G_{v v}$, the forcing term of (13) has to be replaced by the forcing action generated on the structure by the piezo-actuator. The equation governing the dynamic of the plate subject to the moments applied by the piezoactuator can be described by the following PDE [28]:

$$
\rho h \frac{\partial^{2} w}{\partial t^{2}}+D \nabla^{4} w(x, y, t)=\frac{\partial^{2} M_{x}}{\partial x^{2}}+\frac{\partial^{2} M_{y}}{\partial y^{2}},
$$

where the moments per unit length $M_{x}$ and $M_{y}$ represent the forcing term due to the piezoelectric patch (Figure 6).

The forcing term must be defined in order to solve the dynamic equation (17), to make explicit the dependency of the moments on the voltage $V$ and to calculate $G$ and $G_{v v}$.

The flexural moment applied to the structure is due to the deformation of the piezo-patch caused by the voltage
$V$ applied to its terminals. Thus, the expression linking the voltage applied to the terminals of the piezoelectric patch and the moments generated must be derived. For sake of thoroughness, this paper explains the procedure to obtain this term, because for some particular cases (illustrated further on in this section) the available literature does not elucidate the results but is limited to suggesting the procedure to obtain them.

The moments acting on the plate can be described by the following expression:

$$
\begin{aligned}
& M_{p x}=-\int_{A_{x}} z \sigma_{x} d A_{x}, \\
& M_{p y}=-\int_{A_{y}} z \sigma_{y} d A_{y},
\end{aligned}
$$

where $\sigma_{x}$ and $\sigma_{y}$ are the stresses acting on the plate in $x$ and $y$ directions and $A_{x}$ and $A_{y}$ are the transverse cross-sections of the plate (i.e., $A_{x}=b h$ and $A_{y}=a h$ referring to Figure 6).

The system represented in Figure 7, with two colocated piezoelectric actuators and a phase of $180^{\circ}$ between the two of them, shall be discussed now. Relying on the hypothesis of a homogeneous plate, the strain in $x$ and $y$ directions can be considered the same and equals $\varepsilon_{x}=\varepsilon_{y}=\varepsilon=\alpha z$ (see Figure 7). The stresses in the plate and in the piezo-actuators can be expressed as

$$
\begin{aligned}
& \text { Plate }\left\{\begin{array}{l}
\sigma_{x}=\frac{E}{1-\nu^{2}}\left(\varepsilon_{x}+\nu \varepsilon_{y}\right) \\
\sigma_{y}=\frac{E}{1-\nu^{2}}\left(\varepsilon_{y}+\nu \varepsilon_{x}\right)
\end{array} \quad \Longrightarrow \sigma_{x}=\sigma_{y}=\frac{E}{1-v^{2}}(1+\nu) \varepsilon\right. \\
& \text { Piezo }\left\{\begin{array}{l}
\sigma_{x}^{P}=\frac{E_{P}}{1-v_{P}^{2}}\left(\varepsilon_{x}+v_{P} \varepsilon_{y}-\left(1+v_{p}\right) \varepsilon_{P}\right) \\
\widetilde{\sigma}_{x}^{P}=\frac{E_{P}}{1-v_{P}^{2}}\left(\varepsilon_{x}+v_{P} \varepsilon_{y}+\left(1+v_{p}\right) \varepsilon_{P}\right) \\
\sigma_{y}^{P}=\frac{E_{P}}{1-v_{P}^{2}}\left(\varepsilon_{y}+v_{P} \varepsilon_{x}-\left(1+v_{p}\right) \varepsilon_{P}\right) \\
\widetilde{\sigma}_{y}^{P}=\frac{E_{P}}{1-v_{P}^{2}}\left(\varepsilon_{y}+v_{P} \varepsilon_{x}+\left(1+v_{p}\right) \varepsilon_{P}\right),
\end{array}\right.
\end{aligned}
$$

where the symbol $\sim$ refers to the stresses in the actuator at the bottom. In (19) the piezoelectric actuator is assumed to have the same properties in $x$ and $y$ direction, and 


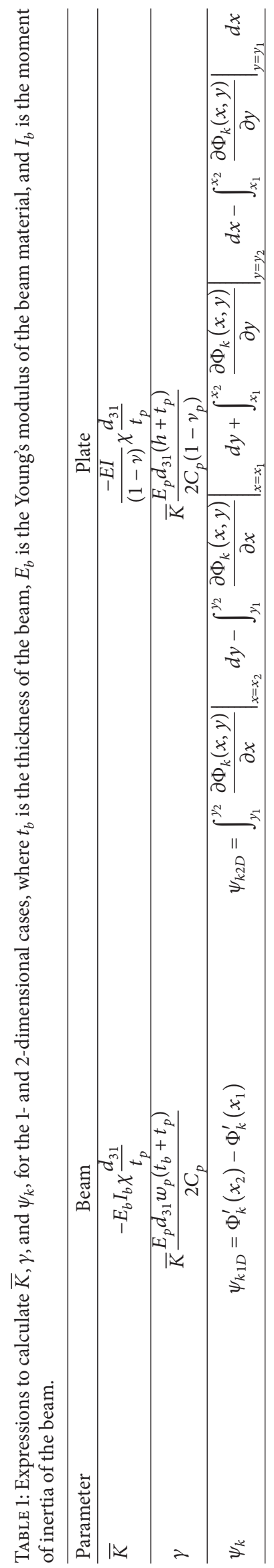




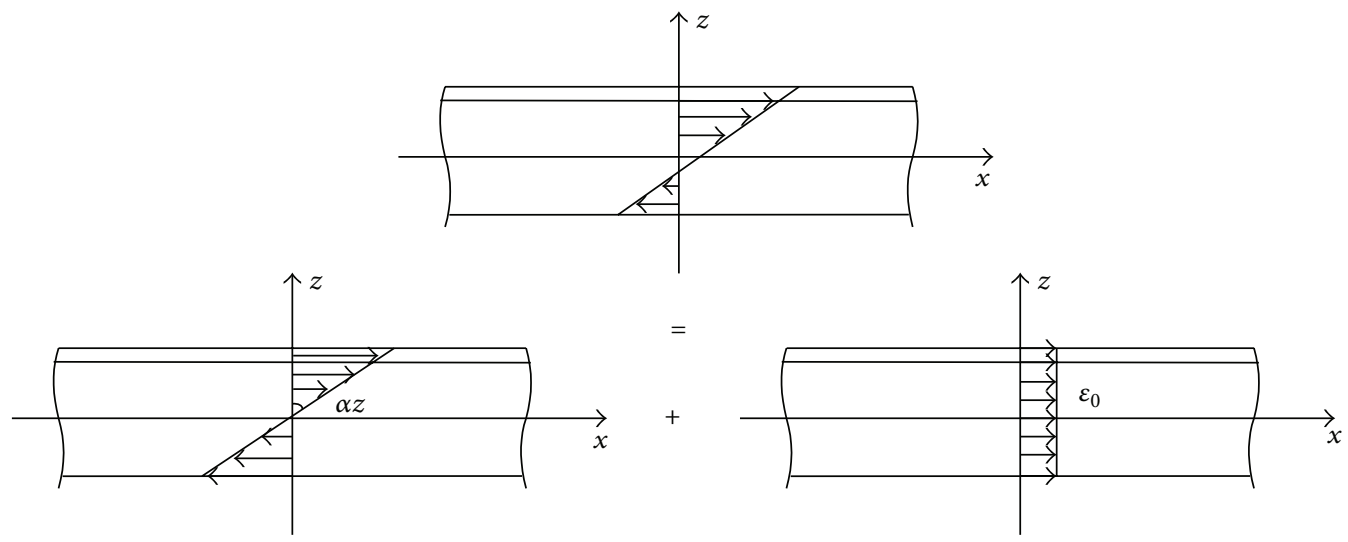

FIGURE 8: Asymmetric strain condition of a plate with a single actuator configuration.

the unconstrained strain of the piezoelectric actuator due to an applied voltage is given by

$$
\varepsilon_{P_{x}}=\varepsilon_{P_{y}}=\varepsilon_{p}=\frac{d_{31}}{t_{p}} V .
$$

The expression of the coefficient $\alpha$, describing the deformation in the plate (Figure 7), must be derived to calculate the analytic expressions of the stresses in the plate, which in turn are necessary to calculate the moments. Such an expression can be computed by applying the moment equilibrium in $x$ and $y$ directions $[3,24,25]$ :

$$
\begin{aligned}
& \int_{-(h / 2)}^{h / 2} \sigma_{x} z d z+\int_{t / 2}^{t / 2+t_{p}} \sigma_{x}^{P} z d z=0, \\
& \int_{-(h / 2)}^{h / 2} \sigma_{y} z d z+\int_{t / 2}^{t / 2+t_{p}} \sigma_{y}^{P} z d z=0 .
\end{aligned}
$$

Just one of the two equilibria can be taken into consideration to derive the expression of $\alpha$, since the plate is assumed to be homogeneous (i.e., $\sigma_{x}=\sigma_{y}=\sigma$ and $\sigma_{x}^{P}=\sigma_{y}^{P}=\sigma^{P}$ ).

Equation (21) shows that the value of the coefficient $\alpha$ changes according to the stresses in the plate (i.e., $\sigma=$ $E \varepsilon=E \alpha z)$. In turn, the stress distribution depends on the layout of the electromechanical structure. The most common configurations for this kind of problem are antisymmetric or asymmetric. The antisymmetric configuration is constituted by two colocated actuators or by colocated sensor-actuator pair. In the antisymmetric configuration instead a single piezo-electric element acting as an actuator is bonded to the structure. The expression of the coefficient $\alpha$ in the asymmetric configuration for a plate has not yet been discussed in its explicit formulation in existing literature; moreover this is the configuration for the plate control by means of shunted piezo-actuators: thus the authors have derived its formulation (see Table 2) and employed it in the herein paper. The strain condition in the plate is asymmetric with this layout and can be decomposed in two parts: flexural and longitudinal (Figure 8 ). The equilibria of moments and axial forces must be imposed to calculate $\alpha$ and $\varepsilon_{0}$; see Figure 8 .
The expressions of $\alpha$ for all the abovementioned configurations, for both beams and plates, are listed in Table 2 .

As for the expressions of $\alpha$ in Table 2, it is opportune to express $\alpha$ as $\alpha=\chi \varepsilon_{p}$, in the following formulations.

The value of $\alpha$ has been derived, so the moment acting on the plate $M_{p x}$ can be computed by substituting (19) into (18):

$$
\begin{aligned}
M_{p x} & =-\int_{A_{x}} z \sigma d A_{x}=-\int_{A_{x}} z \frac{E}{1-v^{2}}(1+\nu) \varepsilon d A_{x} \\
& =-\int_{A_{x}} z \frac{E}{1-v^{2}}(1+\nu) \alpha z d A_{x}=\frac{-E I_{x}}{(1-v)} \chi \varepsilon_{P},
\end{aligned}
$$

where $I_{x}$ is the moment of inertia of the plate $I_{x}=\int_{A_{x}} z^{2} d A_{x}$. The same is valid for the $y$ direction.

The moments per unit length acting on the plate, in directions $x$ and $y$, in (17) are equal (i.e., $M_{x}=M_{y}=M$ ). Thus, the moment per unit length $M$ acting on the plate can be related to the voltage applied to the terminals of the actuator using (20):

$$
M=\frac{-E I}{(1-v)} \chi \frac{d_{31}}{t_{p}} V=\bar{K} V,
$$

where $I=h^{3} /\left[12(1-v)^{2}\right]$.

Having derived the expression of the moment as function of the applied voltage $V$ (23), the expressions of $G$ and $G_{v v}$ can be achieved using (17) and (23) together with a modal coordinate representation in the Laplace domain:

$$
G=\frac{z}{V}=\bar{K} \sum_{k=1}^{\infty} \frac{\Phi_{k}\left(x_{M}\right) \psi_{k}}{s^{2}+2 \xi_{k} \omega_{k} s+\omega_{k}^{2}},
$$

where $\Phi_{k}\left(x_{M}\right)$ represents the value of the $k$ th mode at the measuring point $x_{M}$ :

$$
G_{v v}=\frac{-V_{p}}{V}=\gamma \sum_{k=1}^{\infty} \frac{\psi_{k} \psi_{k}}{s^{2}+2 \xi_{k} \omega_{k} s+\omega_{k}^{2}} .
$$




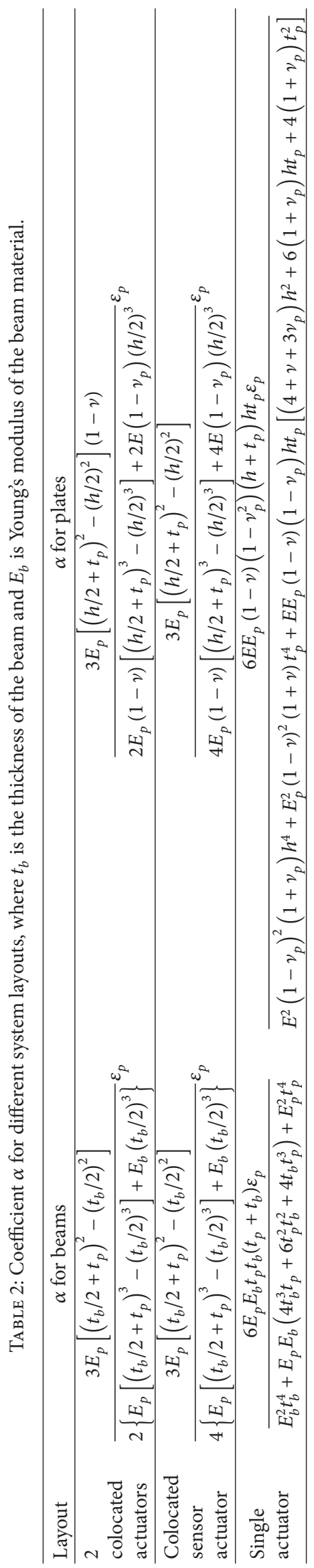


Moreover, $G_{v w}$ in (15) can be expressed as function of the parameters $\gamma$ and $\bar{K}$ shown in Table 1, leading to

$$
G_{v w}=\frac{-V_{p}}{W}=\frac{\gamma}{\bar{K}} \sum_{k=1}^{\infty} \frac{\Phi_{k}\left(x_{F}\right) \psi_{k}}{s^{2}+2 \xi_{k} \omega_{k} s+\omega_{k}^{2}} .
$$

All of the aforementioned expressions can be used for both beams and plates, only differing in the values attributed to the coefficients $\bar{K}, \gamma$, and $\psi_{k}$. These values, for the 1- and 2dimensional cases, are shown in Table 1.

2.4. Formulation of the Frequency Response Function $T_{z w}$. Having defined the transfer functions $G, G_{v v}$, and $G_{v w}$, the frequency response function $T_{z w}$ between the disturbance $W$ and the response $z$ can be calculated by means of (7).

Equation (7) is usually expressed in a slightly different form in literature, indeed using the following formulation:

$$
T_{z w}=\frac{z}{W}=G_{v w} \frac{1}{1+K G_{v v}^{*}} \cdot \frac{1}{G_{v v}} G,
$$

where

$$
\begin{aligned}
G_{v v}^{*} & =\bar{\gamma} \sum_{k=1}^{\infty} \frac{\psi_{k} \psi_{k}}{s^{2}+2 \xi_{k} \omega_{k} s+\omega_{k}^{2}}, \\
\bar{\gamma} & =-\gamma .
\end{aligned}
$$

It is recalled that the terms $\gamma$ and $\psi_{k}$ can be calculated as explained in Table 1 for mono- and bidimensional structures.

The expressions of (7) and (27) are fully coincident. The use of $\bar{\gamma}$ in place of $\gamma$ in (28) is compensated by changing the sign of the term $K G_{v v}^{*}$ in (27). Equation (27) will herein be used from now on in place of (7), for the sake of adhering to the commonplace convention employed in literature.

Relying on the theory described above, an opportune formulation of $T_{z w}$ shall be achieved and employed for all the subsequent calculations and considerations, by virtue of its clarity. The function $T_{z w}$ is the target function for the control strategies that are proposed in the following section. These strategies are based on transfer function considerations and in particular on considerations over the shape of $T_{z w}$ and this allows making full use of the strategies developed for tuning TMDs. In fact, the piezo-actuator shunted to an $R L$ circuit can be considered as the electric equivalent of the TMD [1]. Therefore, tuning strategies similar to those developed for the TMD natural frequency and damping (e.g., [29]) can be used to tune the resistance and inductance of the shunting impedance. Hence, a formulation similar to the one used for TMDs must be derived in order to employ this approach.

The expression of the controller $K$ must be known in order to calculate the transfer function $T_{z w}$ (see (27)). $K$ is represented by the inner loop of the feedback representation (Figure 5) of the controlled plate (5).

$Z$ represents the impedance used to shunt the piezoelectric actuator and in this case it is constituted by the series of a resistance $R$ and an inductance $L$. The differential equation linking the current $i_{z}$ and the voltage $V$ at the piezo-actuator terminals can be expressed as $R i_{z}+L\left(d i_{z} / d t\right)=V$. This equation can be rearranged in the Laplace domain as $V=$ $Z i_{z}=(L s+R) i_{z}$.

Equation (5) thus can be formulated as

$$
K=\frac{s C_{p}(L s+R)}{1+s C_{p}(L s+R)} .
$$

The expression of $K$ should be defined in terms of the electrical frequency $\omega_{p}$ (i.e., the eigenfrequency of the electrical circuit) and of the electrical damping $\xi_{p}$ (i.e., the nondimensional damping ratio of the electrical circuit), so to achieve an expression of $T_{z w}$ similar to those used for TMD systems [29]. Therefore, it is opportune to define the electrical damping $d_{i}$ and the electrical frequency $\omega_{p}$ as

$$
\begin{aligned}
& \omega_{p}=\frac{1}{\sqrt{L C_{p}}}, \\
& \xi_{p}=\frac{R}{2} \sqrt{\frac{C_{p}}{L}} .
\end{aligned}
$$

The controller $K$ can be expressed as a function of these two quantities, substituting (31) and (32) into (30):

$$
K=\frac{s\left(s+2 \xi_{p} \omega_{p}\right)}{s^{2}+2 \xi_{p} \omega_{p} s+\omega_{p}^{2}} .
$$

Concerning single degree-of-freedom systems, the transfer function between the disturbance $W$ and the displacement $z$ can be derived by substituting (24), (28), (26), and (33) into (27):

$$
\begin{aligned}
T_{z w} & =\Phi_{k}\left(x_{M}\right) \Phi_{k}\left(x_{F}\right) \\
\cdot & \frac{s^{2}+2 \xi_{p} \omega_{p} s+\omega_{p}^{2}}{\left(s^{2}+2 \xi_{p} \omega_{p} s+\omega_{p}^{2}\right)\left(s^{2}+2 \xi_{k} \omega_{k} s+\omega_{k}^{2}\right)+\bar{\gamma} \psi_{k}^{2} s\left(s+2 \xi_{p} \omega_{p}\right)} .
\end{aligned}
$$

This formulation is valid for both beams and plates and can be used for any of the configurations listed in Table 2, simply by choosing the appropriate expressions for $\bar{\gamma}$ and $\psi_{k}$. Therefore, the optimization criteria proposed in Section 3 take on general validity and can be used regardless of the configuration and the type of structure. Moreover, the formulation of (34) presents two advantages over the formulation function of $R$ and $L$. The first one is that this formulation allows making use of the tuning formulations developed for TMDs. The second is that it simplifies significantly the mathematical treatment developed in Section 3, used to derive the optimal values of $R$ and $L$.

It is worth remarking that this kind of approach (summarized in (24), (25), (26), and (34)) can be easily extended to the case in which more than one actuator is used. The presence of several actuators, each shunted to an impedance $Z$, can be accounted for adopting a vector formulation for $\psi_{k}$ :

$$
\begin{gathered}
\text { single actuator } \psi_{k} \\
\Longrightarrow n \text { actuators } \bar{\psi}_{k}=\left[\psi_{k_{1}}, \psi_{k_{2}}, \ldots, \psi_{k_{n}}\right] .
\end{gathered}
$$


If the actuators involved in the control of the structure are not identical (i.e., they have different geometrical and electrical characteristics), the parameters $\bar{\gamma}$ and $\bar{K}$ must be modified according to each actuator.

These characteristics make this a particularly efficient approach, with an extended generality and suitable for treating a wide range of problems: the elastic structures taken in consideration can be either a beam or a plate indifferently and the control can be implemented by one or more actuators.

\section{3. $R L$ Tuning Strategies for Damped Elastic Structures}

Closed analytic formulas for tuning the resistance and the inductance of the shunting impedance are presented in this section. These tuning methodologies are based on transfer function considerations and exploit TMD theory. In Section 3.1 the optimal values of the electric eigenfrequency $\omega_{p}$ and the inductance $L$ (31) are derived. Subsequently, Section 3.2 presents three different tuning strategies for the electrical damping $\xi_{p}$ and the resistance $R$.

3.1. Tuning Strategy for the Electrical Eigenfrequency. The tuning method implemented to derive the optimal electrical eigenfrequency $\omega_{p}$ is based on a well-known criterion for undamped structures. Indeed two specific frequencies for undamped structures can be reckoned: one higher and another lower than the mechanical resonance (resp., $\omega_{A}$ and $\left.\omega_{B}\right)$. The behaviour of $\left|T_{z w}\right|$ is independent of the damping factor of the electrical circuit $\xi_{p}$, at the two frequency values $\omega_{A}$ and $\omega_{B}$ (Figure 9(a)). The optimal value of $\omega_{p}$ is found by imposing the same dynamic amplification modulus at these two frequencies [29]:

$$
\left|T_{z w}\right|_{\omega_{A}}=\left|T_{z w}\right|_{\omega_{B}}
$$

This kind of method assumes to be working with an undamped elastic structure. In the case of a damped structure, there is not a single intersection point between curves with different damping values (Figure 9(b)). Nevertheless, the use of frequencies $\omega_{A}$ and $\omega_{B}$ guarantees low approximation: because the frequency values of the intersection points of the different curves fall within limited frequency bands and do not differ greatly from $\omega_{A}$ and $\omega_{B}$, respectively (Figure 9(b)). Furthermore, this optimization strategy was compared against a numerical optimization strategy in which the optimal values for $\omega_{p}$ and $\xi_{p}$ were found numerically by minimizing the maximum value of $\left|T_{z w}\right|_{\xi_{k} \neq 0}$. The results provided by the strategy herein proposed do not differ significantly from the optimization values found by numerical minimization (see Section 4): therefore the hypothesis of undamped elastic structure for tuning the electrical frequency does not introduce noteworthy approximations.

Therefore, the dynamic amplification modulus at $\omega_{A}$ and $\omega_{B}$ must be known, in order to find the optimum value of $\omega_{p}$. It is noteworthy that $\left|T_{z w}\right|$ is independent of the value of $\xi_{p}$ at these two frequencies, and therefore the response at these two

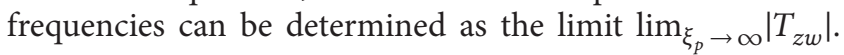
Relying on (34)

$$
\begin{aligned}
\left|T_{z w}\right|_{\omega_{A}, \omega_{B}} & =\lim _{\xi_{p} \rightarrow \infty}\left|\Phi_{k}\left(x_{M}\right) \Phi_{k}\left(x_{F}\right) \frac{-\omega^{2}+2 i \xi_{p} \omega_{p} \omega+\omega_{p}^{2}}{\left(-\omega^{2}+2 i \xi_{p} \omega_{p} \omega+\omega_{p}^{2}\right)\left(-\omega^{2}+2 i \xi_{k} \omega_{k} \omega+\omega_{k}^{2}\right)+\bar{\gamma} \psi_{k}^{2} i \omega\left(i \omega+2 \xi_{p} \omega_{p}\right)}\right| \\
& = \pm\left|\Phi_{k}\left(x_{M}\right) \Phi_{k}\left(x_{F}\right)\right| \frac{1}{\left(-\omega^{2}+\omega_{k}^{2}+\bar{\gamma} \psi_{k}^{2}\right)},
\end{aligned}
$$

where $\omega$ represents the circular frequency and $i$ is the imaginary unit. $\omega$ must be fixed to $\omega_{A}$ and $\omega_{B}$ in (37) and the + and - signs are referred to the value of $\omega_{A}$ and $\omega_{B}$, respectively. Moreover (37) is yielded considering $\xi_{k}=0$.

Hence, the substitution of (37) in (36) leads to

$$
\frac{1}{\left(-\omega_{A}^{2}+\omega_{k}^{2}+\bar{\gamma} \psi_{k}^{2}\right)}=\frac{-1}{\left(-\omega_{B}^{2}+\omega_{k}^{2}+\bar{\gamma} \psi_{k}^{2}\right)} .
$$

And the following expression is achieved by rearranging (38):

$$
\omega_{A}^{2}+\omega_{B}^{2}=2 \omega_{k}^{2}+2 \psi_{k}^{2} \bar{\gamma}
$$

Furthermore (34) can be expressed (neglecting the structural damping $\xi_{k}$ ) as

$$
T_{z w_{\xi_{k}=0}}=\Phi_{k}\left(x_{M}\right) \Phi_{k}\left(x_{F}\right) \frac{A+2 i \xi_{p} B}{C+2 i \xi_{p} D}
$$

where $A, B, C$, and $D$ are

$$
\begin{aligned}
& A=-\omega^{2}+\omega_{p}^{2}, \\
& B=\omega_{p} \omega, \\
& C=\left(-\omega^{2}+\omega_{p}^{2}\right)\left(-\omega^{2}+\omega_{k}^{2}\right)-\psi_{k}^{2} \bar{\gamma} \omega^{2}, \\
& D=\omega_{p} \omega\left(-\omega^{2}+\omega_{k}^{2}+\psi_{k}^{2} \bar{\gamma}\right) .
\end{aligned}
$$

As highlighted by Krenk in [29], the dynamic amplification modulus at $\omega_{A}$ and $\omega_{B}$ is independent of the damping, and this can be mathematically expressed as

$$
\frac{A^{2}}{C^{2}}=\frac{B^{2}}{D^{2}} \Longrightarrow A D= \pm B C .
$$




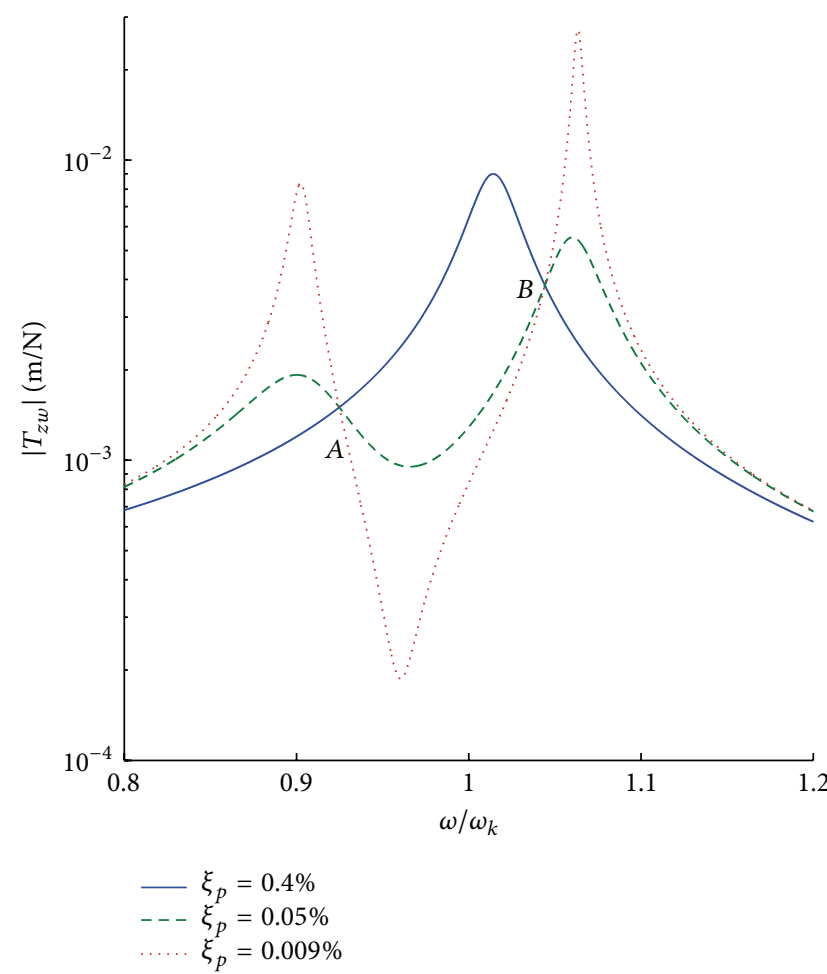

(a)

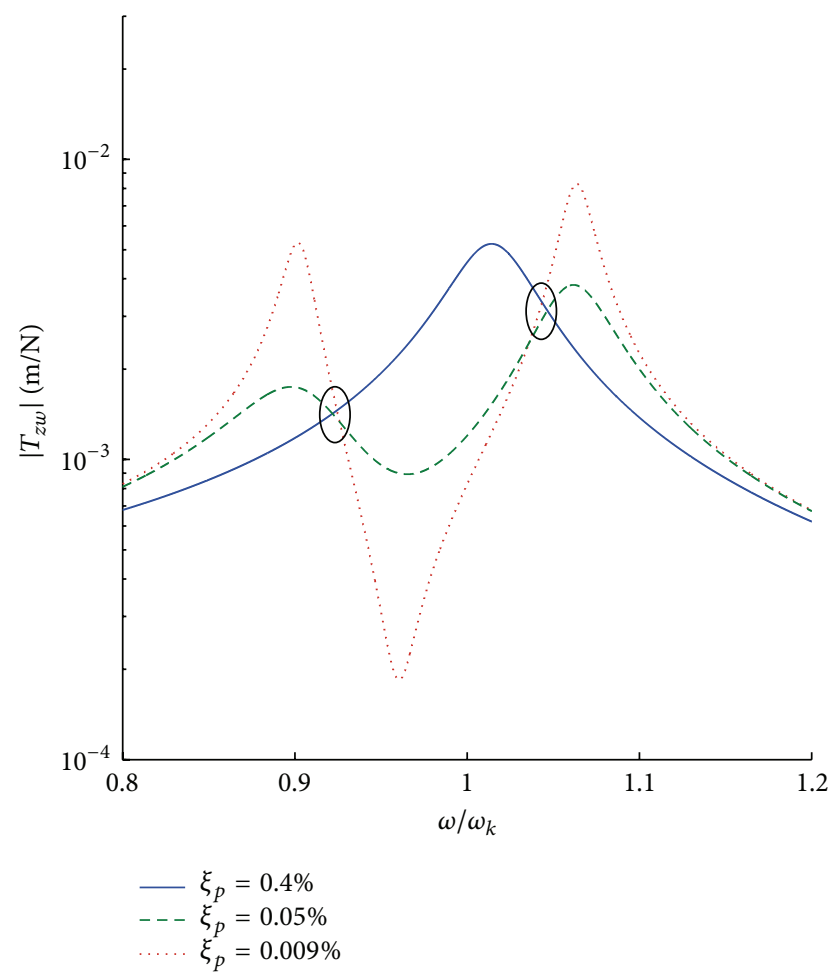

(b)

FIGURE 9: $\left|T_{z w}\right|$ for (a) undamped elastic structure and (b) damped elastic structure: $\xi_{k}=1 \%$.

Substituting $A, B, C$, and $D$ in (42) gives

$$
\begin{aligned}
& \left(-\omega^{2}+\omega_{p}^{2}\right) \omega_{p} \omega\left(-\omega^{2}+\omega_{k}^{2}+\psi_{k}^{2} \bar{\gamma}\right) \\
& \quad= \pm \omega_{p} \omega\left(\left(-\omega^{2}+\omega_{p}^{2}\right)\left(-\omega^{2}+\omega_{k}^{2}\right)-\psi_{k}^{2} \bar{\gamma} \omega^{2}\right) .
\end{aligned}
$$

The use of the + sign leads to the trivial solution $\omega=0$, where there is no motion and therefore no damping force. The minus sign instead gives the following relation:

$$
\omega_{A}^{2}+\omega_{B}^{2}=\omega_{k}^{2}+\psi_{k}^{2} \bar{\gamma}+\omega_{p}^{2}
$$

Then, the following expression is achieved by substituting (39) in (44):

$$
\omega_{p}=\sqrt{\omega_{k}^{2}+\psi_{k}^{2} \bar{\gamma}}
$$

This formulation allows calculating the optimum value of $\omega_{p}$.

Finally, if we consider that the electrical frequency can be expressed as a function of the inductance of the shunting circuit by using (31), the optimal value of $L$ is

$$
L=\frac{1}{C_{p} \omega_{p}^{2}}=\frac{1}{C_{p}\left(\omega_{k}^{2}+\psi_{k}^{2} \bar{\gamma}\right)} .
$$

3.2. Tuning Strategies for the Electrical Damping Ratio. Three strategies have been developed to tune the value of the nondimensional damping ratio $\xi_{p}$ (and thus the value of the resistance $R$ ) and are designed allowing for damped elastic structures, which are seldom accounted for in literature. The first two methodologies discussed here are based on standard tuning criteria $([29,30])$ for the TMD devices, while the third one is based on considerations on the shape of $T_{z w}$ as function of the electrical damping $\xi_{p}$. The tuning criteria listed below are explained in Figure 10 and described in detail in Sections 3.2.1, 3.2.2, and 3.2.3:

(1) Optimization 1: $\left|T_{z w}\right|_{\omega_{p}, \xi_{k \neq 0}}=\left|T_{z w}\right|_{\omega_{A}, \xi_{k \neq 0}}$,

(2) Optimization 2: $\left.\left(\partial\left|T_{z w}\right|_{\xi_{k \neq 0}} / \partial \omega\right)\right|_{\omega_{A}}=0$,

(3) Optimization 3: $\partial\left|T_{z w}\right|_{\omega_{A}, \xi_{k \neq 0}} / \partial \xi_{p}=0$.

3.2.1. Optimization 1. The first optimization criterion proposed for the electrical damping makes use of the procedure developed by Krenk for TMDs [29]. The optimal value of the damping $\xi_{p}$ is found by imposing equal dynamic amplification $\left|T_{z w}\right|$ at two different frequencies: $\omega_{A}$ and a frequency given by the square root of the arithmetic mean between $\omega_{A}^{2}$ and $\omega_{B}^{2}$. This frequency is found to be equal to the electrical frequency $\omega_{p}$. Indeed, relying on (44) and (45), the following expression is yielded:

$$
\frac{\omega_{A}^{2}+\omega_{B}^{2}}{2}=\omega_{k}^{2}+\psi_{k}^{2} \bar{\gamma}=\omega_{p}^{2} .
$$

Unlike the procedure proposed by Krenk [29], the authors of this paper have decided to reckon with damped systems, in order to reach a formulation that can account for the whole dynamic behaviour of the system. 


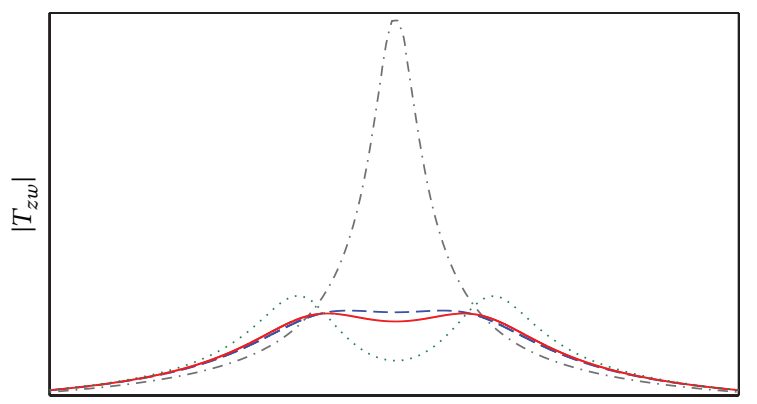

Frequency

-.. Mechanical system

- - - Optimisation 1

- Optimisation 2

..... Optimisation 3

Figure 10: Example of the three optimization criteria for the electrical damping $\xi_{p}$ for a generic system.

The dynamic amplification value at $\omega_{A}$ and $\omega_{B}$ must be calculated to find the value for $\xi_{p}$. Its expression can be derived by substituting the expression of $\omega_{p}$ (45) in (43) with negative sign and solving it with respect to $\omega_{A}$ and $\omega_{B}$ :

$$
\omega_{A / B}=\sqrt{\frac{2\left(\omega_{k}^{2}+\psi_{k}^{2} \bar{\gamma}\right) \mp \sqrt{2 \psi_{k}^{2} \bar{\gamma}\left(\psi_{k}^{2} \bar{\gamma}+\omega_{k}^{2}\right)}}{2}},
$$

where $\omega_{A}$ is obtained using the negative sign, while $\omega_{B}$ the positive.

Rearranging (34), $T_{z w}$ can be expressed as

$$
T_{z w}=\Phi_{k}\left(x_{M}\right) \Phi_{k}\left(x_{F}\right) \frac{A_{1}+i B_{1}}{C_{1}+i D_{1}},
$$

where $A_{1}, B_{1}, C_{1}$, and $D_{1}$ are

$$
\begin{aligned}
A_{1} & =-\omega^{2}+\omega_{p}^{2}, \\
B_{1} & =2 \xi_{p} \omega_{p} \omega, \\
C_{1} & =\left(\omega^{4}-\omega_{k}^{2} \omega^{2}-4 \xi_{p} \xi_{k} \omega_{k} \omega_{p} \omega^{2}-\omega_{p}^{2} \omega^{2}+\omega_{k}^{2} \omega_{p}^{2}\right. \\
& \left.-\psi_{k}^{2} \bar{\gamma} \omega^{2}\right), \\
D_{1} & =2 \omega\left(-\xi_{k} \omega_{k} \omega^{2}-\xi_{p} \omega_{p} \omega^{2}+\xi_{p} \omega_{p} \omega_{k}^{2}+\xi_{k} \omega_{k} \omega_{p}^{2}\right. \\
& \left.+\psi_{k}^{2} \bar{\gamma} \xi_{p} \omega_{p}\right) .
\end{aligned}
$$

Therefore, the square of the dynamic amplification modulus can be expressed as a function of the parameters $A_{1}, B_{1}, C_{1}$, and $D_{1}$ :

$$
\left|T_{z w}\right|_{\xi_{k \neq 0}}^{2}=\frac{A_{1}^{2} C_{1}^{2}+B_{1}^{2} D_{1}^{2}+B_{1}^{2} C_{1}^{2}+A_{1}^{2} D_{1}^{2}}{\left(C_{1}^{2}+D_{1}^{2}\right)^{2}} .
$$

The dynamic amplification modulus in $\omega_{A}$ and $\omega_{p}$ can be derived by substituting (47) and (48) in (51). Then, the equation needed to find the electrical damping ratio $\xi_{p}$ can be derived by imposing

$$
\left|T_{z w}\right|_{\omega_{p}, \xi_{k \neq 0}}=\left|T_{z w}\right|_{\omega_{A}, \xi_{k \neq 0}} .
$$

The complete equation, function of the electrical and mechanical parameters, shall not be included in this paper, for sake of conciseness.

Solving (52) with respect to the electrical damping $\xi_{p}$ leads to a sixth-order equation which does not provide a closed analytic formulation for $\xi_{p}$. Nonetheless, the exact values of the electrical damping can be yielded, if all the values of the other variables are known.

On the contrary, a closed analytic formulation can indeed be derived for $\xi_{p}$, in the case of undamped elastic structures. The same procedure described above leads to an electrical damping equal to

$$
\xi_{p, \xi_{k}=0}=\sqrt{\frac{\psi_{k}^{2} \bar{\gamma}}{2 \omega_{p}^{2}}}
$$

3.2.2. Optimization 2. The second optimization criterion for the electrical damping $\xi_{p}$ is based on a standard optimization criterion for the damping element in TMD systems. The optimal value for $\xi_{p}$ is found by setting the maximum value of $\left|T_{z w}\right|$ at $\omega_{A}$. Therefore, the square root of (51) is derived with respect to the frequency $\omega$, and then the resulting function is set equal to zero at $\omega_{A}(48)$ :

$$
\left.\frac{\partial\left|T_{z w}\right| \xi_{k \neq 0}}{\partial \omega}\right|_{\omega_{A}}=0
$$

The optimal value for $\xi_{p}$ is derived by solving (54) with respect to the electrical damping $\xi_{p}$. The complete expression of (54) as function of $\xi_{p}$ is not expounded in this paper because of its complexity and because it is a sixth-order equation in $\xi_{p}$. Therefore, also in this case a closed formulation to derive the electrical damping cannot be achieved.

3.2.3. Optimization 3. Unlike the optimization strategies discussed above, both based on TMD tuning techniques, the third optimisation strategy here discussed originates from considerations on the trend of the dynamic amplification modulus $\left|T_{z w}\right|$ in $\omega_{A}$ and $\omega_{B}$ as function of the electrical damping.

In fact, $\left|T_{z w}\right|_{\omega_{A}, \omega_{B}}$ has a minimum for a specific value of $\xi_{p}$ (Figure 11). Hence, this optimization criterion is based the use of the electrical damping value which minimises the dynamic amplification modulus at $\omega_{A}$. This condition is expressed by the following relation:

$$
\frac{\partial\left|T_{z w}\right|_{\omega_{A}, \xi_{k \neq 0}}}{\partial \xi_{p}}=0,
$$

where $\left|T_{z w}\right|_{\omega_{A}, \xi_{k \neq 0}}$ is found by substituting (45) and (48) in (51). 


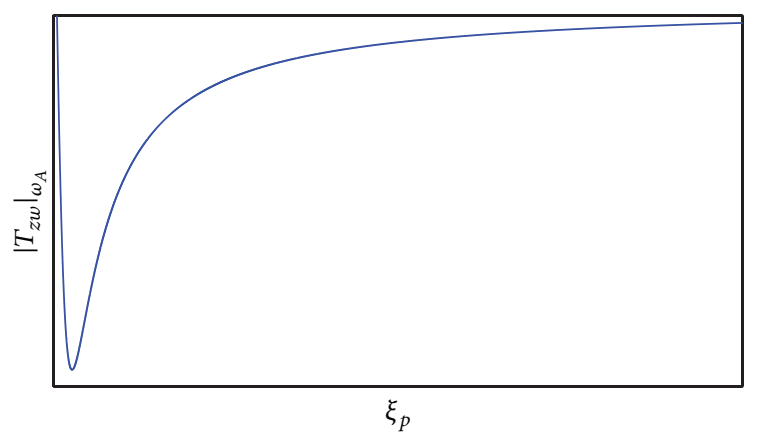

Figure 11: Dynamic amplification modulus at frequency $\omega_{A}$ as function of the electrical damping $\xi_{p}$ for a generic system.

Equation (55) leads to a sixth-order equation in $\xi_{p}$, likewise to (54) and (52).

The three optimization methods for electrical damping discussed in this paper each lead to different values of $\xi_{p}$ and therefore to different shapes of the dynamic amplification modulus $\left|T_{z w}\right|$ (Figure 10). Figure 10 illustrates that the third method generates lower damping values than the first method and that the second strategy instead leads to a behaviour midway between the two. The following sections present performance and robustness analyses of the three optimisation methods, displaying advantages and disadvantages of each strategy, in terms of vibration reduction and robustness against uncertainties on the electrical and mechanical parameters.

\section{Performance Analysis}

Three different tuning methodologies for the shunt impedance parameters are expounded in Section 3. Each of them leads to different values for the resistance $R$ and therefore to different levels of damping; consequently the performance, in terms of vibration attenuation, changes according to the tuning strategy employed. This section deals with the analysis of the performance of these tuning methodologies in the entire domain of application of this type of control method. The domain considered in this analysis is described in Section 4.1: it has been chosen so to take into account the majority of cases possible in actual applications of light and thin structures. Subsequently, in Section 4.2, a comparison is drawn between the optimal damping values required by each of the three tuning strategies and the optimum achieved through a numerical minimization. In addition, a performance analysis in terms of vibration reduction is discussed in Section 4.3, showing the effectiveness of each of the tuning strategies in the whole domain analysed. All of these analyses have also been carried out on two additional tuning strategies: optimisation 1 and optimisation 2 in the case of no structural damping (i.e., carrying out all the calculations to obtain the value of $\xi_{p}$, considering a null mechanical damping, e.g., (53), and then applying the solution found to a mechanical system with a nonnull mechanical damping). Finally, the effect of the parameter $\psi_{k}^{2} \bar{\gamma}$ on the performance of the control system is studied in detail and explained in Section 4.4.

4.1. Domain Description. A wide application domain is taken into consideration in the study of the behaviour of the tuning strategies analysed here in order to make this study as general as possible. Cases taken into consideration include extreme situations, their opposites, and the range of intermediate ones, so as to account for the majority of actual real applications involving shunted piezoelectric actuator controls: highly flexible and extremely rigid structures, very high and very low natural frequencies to be controlled, best and worst position of the piezo-actuator for controlling a given mode (i.e., $\psi_{k}$ ), and different geometries and materials of the elastic structure and of the actuator. Of course, this approach introduces even near-implausible situations into the analysis, but it does allow generalising the conclusions arising from the analysis and to exclude the eventuality of different behaviours for test cases not taken into consideration. The range of values considered for each parameter is shown in Table 3, where the values of $\bar{\gamma}$ were derived from the geometrical and material characteristics according to the formulas of Table 1 and for different kinds of constraint. Equations (45), (52), (53), (54), and (55) show that the shunting impedance parameters (i.e., $\omega_{p}$ and $\xi_{p}$ ) and the dynamic amplification $T_{z w}$ (34) depend solely on the problem parameters in Table 3 . Thus, all the cases included in the domain described by the quantities in Table 3 can be represented by modifying the problem parameters $\psi_{k}^{2} \bar{\gamma}, \omega_{k}$, and $\xi_{k}$. Each of these parameter was altered by increments of $50 \mathrm{rad}^{2} / \mathrm{s}^{2}, 100 \cdot 2 \pi \mathrm{rad} / \mathrm{s}$ (i.e., $100 \mathrm{~Hz}$ ), and $0.0005 \%$, respectively, and a simulation was performed for each combination.

All the results of the simulations (see Section 4.2) showed a monotonic trend with respect to the three problem parameters $\psi_{k}^{2} \bar{\gamma}, \omega_{k}$, and $\xi_{k}$; hence only a selection of representative cases is reported in this paper, also for sake of conciseness. Three values of $\psi_{k}^{2} \bar{\gamma}, \omega_{k}$, and $\xi_{k}$ have been selected: corresponding to a high, medium, and low level of the parameters, respectively (see Table 4). This approach leads to the analysis of 27 cases, numbered 1 to 27 (Table 5). The rationale behind the selection of the values in Table 5 shall be clarified further on in this paper.

4.2. Comparison of the Tuning Methods. Having defined the domain to consider in the analysis, the optimisation methods under consideration can be compared to a reference method taken as the optimum. In this case the reference method is a numeric minimisation of the maximum value of the dynamic amplification modulus $\left|T_{z w}\right|_{\max }$. In fact, the abovementioned optimisation methods rely on some simplifications (e.g., the use of $\omega_{A}$ and $\omega_{B}$, which do not actually exist in case of nonnull mechanical damping) and the numerical minimisation acts as a reference to check their reliability. The comparison takes into consideration two reference quantities, representative of the effectiveness of the control system: the maximum value of the dynamic amplification modulus $\left|T_{z w}\right|_{\max }$ and the value of $\left|T_{z w}\right|$ at the natural frequency $\omega_{k}$. The latter is representative of 


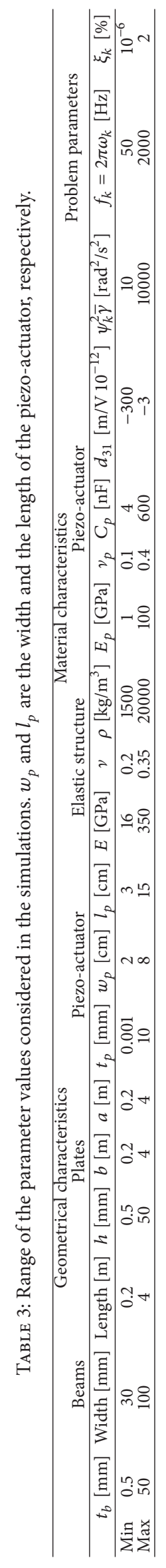


TABLE 4: Selected values for $\psi_{k}^{2} \bar{\gamma}, \omega_{k}$, and $\xi_{k}$.

\begin{tabular}{lccc}
\hline Parameter & Low value & $\begin{array}{c}\text { Medium } \\
\text { value }\end{array}$ & High value \\
\hline$\psi_{k}^{2} \bar{\gamma}\left[\mathrm{rad}^{2} / \mathrm{s}^{2}\right]$ & 10 & 1010 & 1960 \\
$\omega_{k}[\mathrm{rad} / \mathrm{s}]$ & $200 \cdot 2 \pi$ & $500 \cdot 2 \pi$ & $1000 \cdot 2 \pi$ \\
$\xi_{k}[\%]$ & 0.0001 & 0.05 & 0.2 \\
\hline
\end{tabular}

the behaviour of the control system in the case of monoharmonic excitation at the natural frequency $\omega_{k}$. The former instead is representative of the maximum dynamic amplification in case of a wideband random excitation around the natural frequency $\omega_{k}$. The indexes used to represent the comparison are expressed in decibels as

$$
\begin{aligned}
\varepsilon_{\max } & =20 \log _{10} \frac{\left|T_{z w}\right|_{\text {max,num }}}{\left|T_{z w}\right|_{\text {max }, \text { ott }_{x}}}, \\
\varepsilon_{\omega_{k}} & =20 \log _{10} \frac{\left|T_{z w}\right|_{\omega_{k}, \text { num }}}{\left|T_{z w}\right|_{\omega_{k}, \text { ott }_{x}}},
\end{aligned}
$$

where the subscript num refers to the numerical optimization and the subscript ott $x$ refers to the optimization method $x$ (e.g., optimisations 1, 2, etc.).

As mentioned before, two additional tuning methodologies were included in this analysis, because of their ease of use: optimization 1 in the case of no structural damping (53) and the equivalent of the optimization 2 for undamped elastic structures, obtained through an empirical procedure developed in [10] (these methodologies will be named 1-und and 2 -und, resp.). The optimal values for $\xi_{p}$ and $\omega_{p}$ in the case of method 2-und are expressed by the following formulations [10]:

$$
\begin{aligned}
& \xi_{p}=\frac{0.63}{\omega_{k}}\left(\psi_{k}^{2} \bar{\gamma}\right)^{0.495}, \\
& \omega_{p}=\frac{0.495}{\omega_{k}} \psi_{k}^{2} \bar{\gamma}+\omega_{k} .
\end{aligned}
$$

These two methods were included in the analysis because they are based on closed formulas that are easy to employ.

Before analysing the results in terms of the indexes of (56), a comparison between the values of the electrical frequency and of the electrical damping, achieved by the different methods, is shown in Figures 12 and 13, respectively. Figure 12 shows that the electrical frequency obtained by the numerical minimisation is near the same of those with the other methods (i.e., all the lines of the plot are almost superimposed). As for the electrical damping $\xi_{p}$, its trend with respect to the problem parameters is shown in Figure 13. As expected, the electrical damping values obtained by optimisation 1-und (53) and 2-und (57) do not change when changing the structural damping $\xi_{k}$. Moreover, also the optimisation method 3 leads to damping values constant with varying $\xi_{k}$. As for optimisations 1 and 2 and the numerical optimum, the electrical damping increases with the increase of the structural damping. On the contrary, the trend of $\xi_{p}$

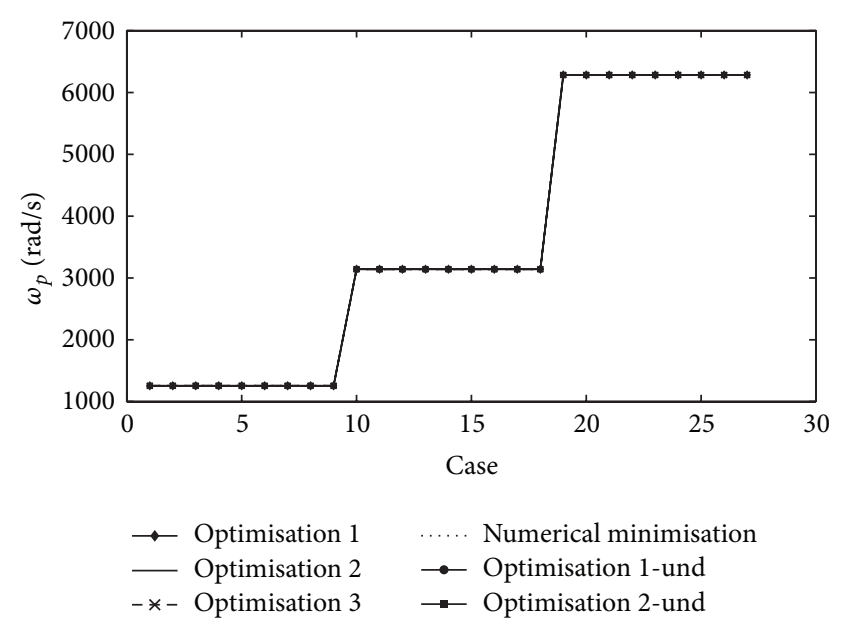

FIGURE 12: $\omega_{p}$ values for the different optimisation methods considered and for all the cases of Table 5.



FIGURE 13: $\xi_{p}$ values for the optimisation methods considered and for all the cases shown in Table 5.

with respect to $\omega_{k}$ and $\psi_{k}^{2} \bar{\gamma}$ is the same for all the methods considered: the electrical damping $\xi_{p}$ increases with the increase of $\psi_{k}^{2} \bar{\gamma}$ and decreases with the increase of $\omega_{k}$. It is worth noting that the optimisation methods overall can be divided into three groups, according to the damping level achieved. Optimisations 1 and 1-und provide similar results and differ only at high structural damping levels. Moreover, these two among all the methods considered lead to the highest level of damping. Optimisations 2, 2-und, and the numerical one provide similar damping values but lower than optimisations 1 and 1-und. Finally, optimisation 3 behaves wholly differently: providing the lowest level of damping. This peculiarity of optimisation 3 and the difference from optimisations 1 and 2 in terms of $\xi_{p}$ can already be inferred from Figure 10: the high damping provided by optimisation 1 flattens out the dynamic amplification modulus in the area around the resonance; conversely, the lower damping of optimisation 3 generates overshoots before and after the resonance and a dynamic amplification modulus at resonance 
TABLE 5: Case description.

\begin{tabular}{lccccccccc}
\hline & & $\psi_{k}^{2} \bar{\gamma}_{\text {low }}$ & & & $\psi_{k}^{2} \bar{\gamma}_{\text {medium }}$ & & $\psi_{k}^{2} \bar{\gamma}_{\text {high }}$ & \\
& $\xi_{k_{\text {low }}}$ & $\xi_{k_{\text {medium }}}$ & $\xi_{\text {high }_{\text {high }}}$ & $\xi_{\text {low }_{\text {low }}}$ & $\xi_{k_{\text {medium }}}$ & $\xi_{k_{\text {high }}}$ & $\xi_{k_{\text {low }}}$ & $\xi_{k_{\text {medium }}}$ & $\xi_{k_{\text {high }}}$ \\
\hline$\omega_{k_{\text {low }}}$ & 1 & 2 & 3 & 4 & 5 & 6 & 7 & 8 & 9 \\
$\omega_{k_{\text {medium }}}$ & 10 & 11 & 12 & 13 & 14 & 15 & 16 & 17 & 18 \\
$\omega_{k_{\text {high }}}$ & 19 & 20 & 21 & 22 & 23 & 24 & 25 & 26 & 27 \\
\hline
\end{tabular}

considerably lower than in the other cases. Optimisation 2 displays a behaviour midway between methods 1 and 3 .

As for the performance comparison in terms of $\varepsilon_{\max }(56)$, the numerical minimisation shows a better behaviour than methods 1, 2, 1-und, and 2-und but $\left|\varepsilon_{\max }\right|$ never exceeds $0.3 \mathrm{~dB}$. The only method with a different behaviour is optimisation method 3 , for which $\varepsilon_{\max }$ varies from $-2 \mathrm{~dB}$ for low mechanical damping values to $-1.2 \mathrm{~dB}$ for high structural damping values. These values make for a similar behaviour of all the methods when taking into consideration the maximum value of the dynamic amplification magnitude.

As for $\varepsilon_{\omega_{k}}$, the numerical optimisation behaves rather alike to optimisations 1, 1-und, 2, and 2-und (maximum value of $\left|\varepsilon_{\omega_{k}}\right|$ equal to $1.5 \mathrm{~dB}$ ). On the other hand, optimisation 3 behaves considerably better than the others on account of the lower damping level introduced in the system, as expected. The index $\varepsilon_{\omega_{k}}$ varies from $6.2 \mathrm{~dB}$ for low mechanical damping to $4 \mathrm{~dB}$ with high structural damping. All of these aspects can also be inferred from Figures 14 and 15, as will be discussed in the following section.

4.3. Performance Analysis. The previous subsection shows that all the methods have approximately the same behaviour, except the optimisation method 3 which features superior results when taking into consideration the value of $\left|T_{z w}\right|$ at resonance. Otherwise, considering the performances in terms of $\left|T_{z w}\right|_{\max }$, all of the methods can be expected to behave alike. The performances of each method are calculated as the reduction of $\left|T_{z w}\right|$ with respect to the uncontrolled structure at the electric resonance and at the maximum of $\left|T_{z w}\right|:$

$$
\begin{gathered}
\operatorname{Perf}\left|T_{z w}\right|_{\omega_{k}}=20 \log _{10} \frac{\left|T_{z w}\right|_{\omega_{k}, \mathrm{nc}}}{\left|T_{z w}\right|_{\omega_{k}, x}}, \\
\operatorname{Perf}\left|T_{z w}\right|_{\text {max }}=20 \log _{10} \frac{\left|T_{z w}\right|_{\text {max }, \mathrm{nc}}}{\left|T_{z w}\right|_{\text {max }, x}},
\end{gathered}
$$

where the subscript $\mathrm{nc}$ is referred to the uncontrolled structure, and the subscript $x$ to the optimisation method considered.

As expected, Figures 14 and 15 confirm the considerations made in Section 4.2. The value of this performance analysis lies in the possibility of determining the performance trends as functions of the three problem parameters: $\omega_{k}, \xi_{k}$, and $\psi_{k}^{2} \bar{\gamma}$. Figures 14 and 15 show that the performances of all the methods improve with the increase of $\psi_{k}^{2} \bar{\gamma}$, while they worsen with the increase of the natural frequency and the damping of the elastic structure. The effect of the structural

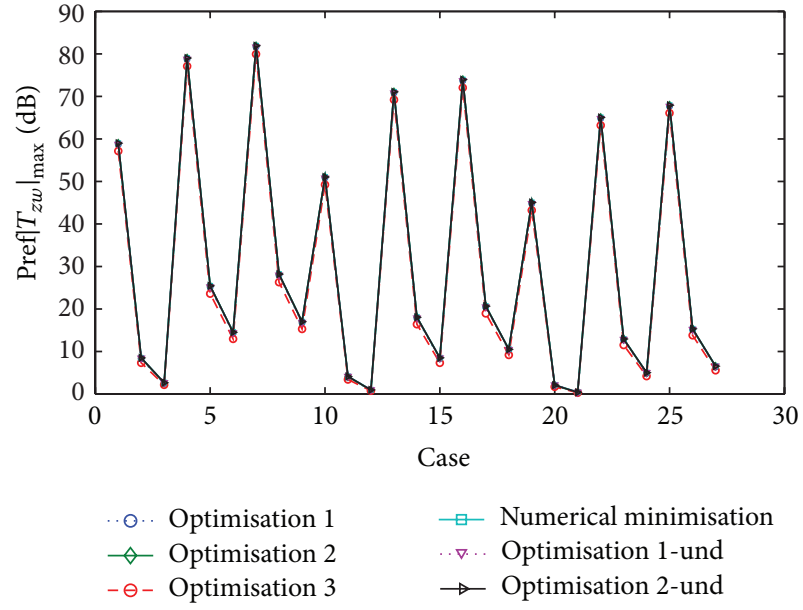

FIgURE 14: Perf $\left|T_{z w}\right|_{\max }$ for all the methods considered and for all the cases listed in Table 5.

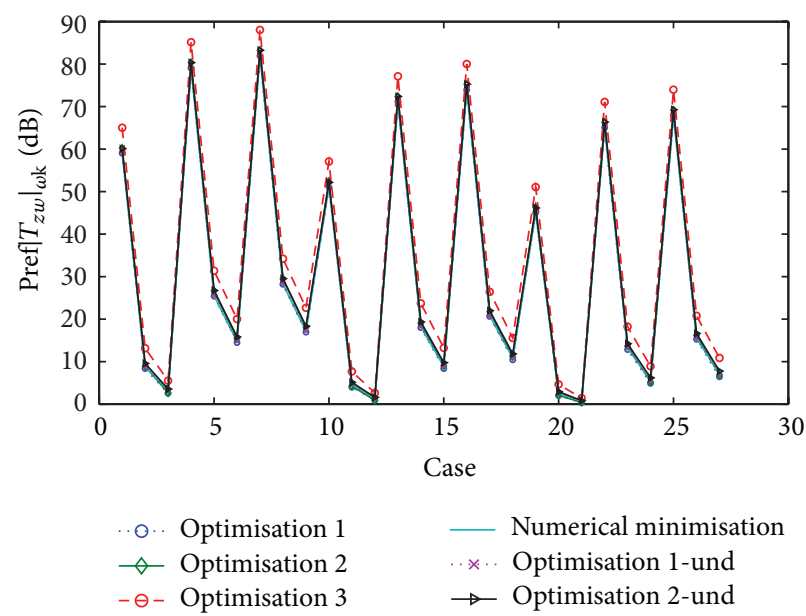

FIGURE 15: Perf $\left|T_{z w}\right|_{\omega_{k}}$ for all the methods considered and for all the cases listed in Table 5 .

damping is consistent with the expected results: the structural damping reduces the vibration of the mechanical system, so consequently the effect that the shunt system is capable of supplementing is limited. The mechanical energy available to be converted into electrical energy is indeed limited and the control action is less effective than in the case of structures with low damping values. It should be remarked that this effect, together with the reduction of performances consistent with the increase of $\omega_{k}$, limits the range of applicability of this kind of control system. Figures 16, 17, and 18 illustrate the 


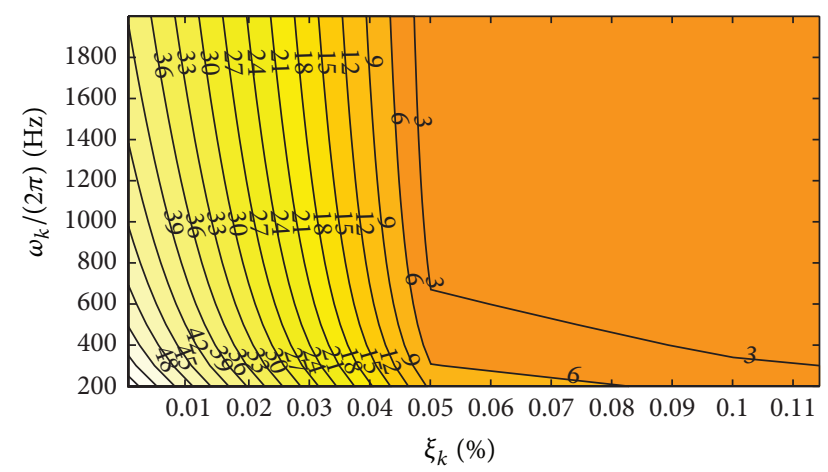

FIgURE 16: $\operatorname{Perf}\left|T_{z w}\right|_{\max }$ as function of $\xi_{k}$ and $\omega_{k}$ for $\psi_{k}^{2} \bar{\gamma}_{\text {low }}$ (see Table 4).

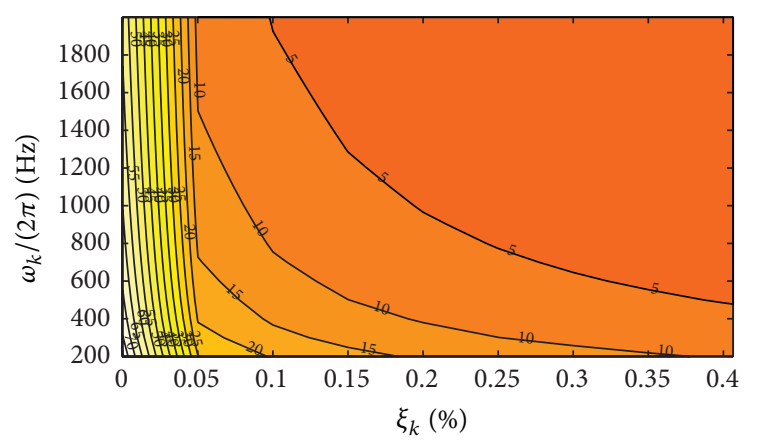

Figure 17: Perf $\left|T_{z w}\right|_{\max }$ as function of $\xi_{k}$ and $\omega_{k}$ for $\psi_{k}^{2} \bar{\gamma}_{\text {medium }}$ (see Table 4).

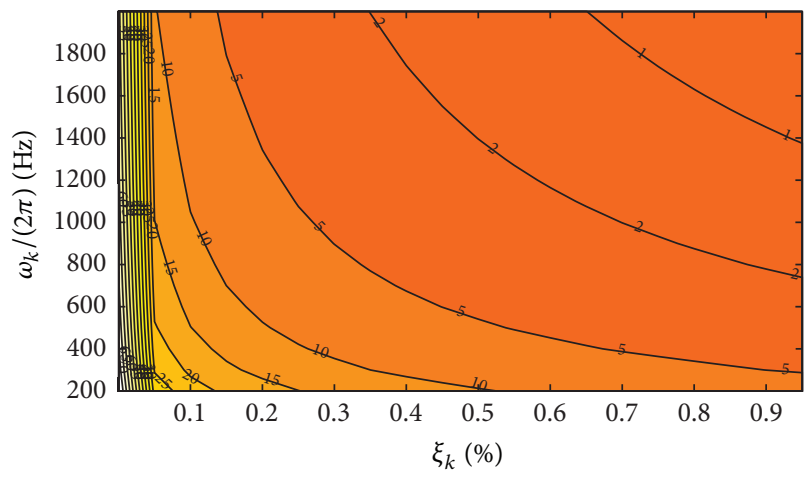

FIgURE 18: Perf $\left|T_{z w}\right|_{\max }$ as function of $\xi_{k}$ and $\omega_{k}$ for $\psi_{k}^{2} \bar{\gamma}_{\text {high }}$ (see Table 4).

effect of $\xi_{k}$ and $\omega_{k}$ on the efficacy of this kind of control system, in the case of optimization 1 . The cases expressed in these figures demonstrate the effect of these two parameters in all situations, whatever optimisation algorithm is used. It may be observed that the effectiveness of the control decreases with the increase of the damping and the natural frequency of the structural mode. The maximum values of $\xi_{k}$ and $\omega_{k}$ providing satisfactory performances depend on the value of $\psi_{k}^{2} \bar{\gamma}$ : when $\psi_{k}^{2} \bar{\gamma}$ is low the effectiveness of the control strategy is drastically curtailed, for damping values higher than $0.05 \%$ and eigenfrequencies higher than $300 \mathrm{~Hz}$. These threshold

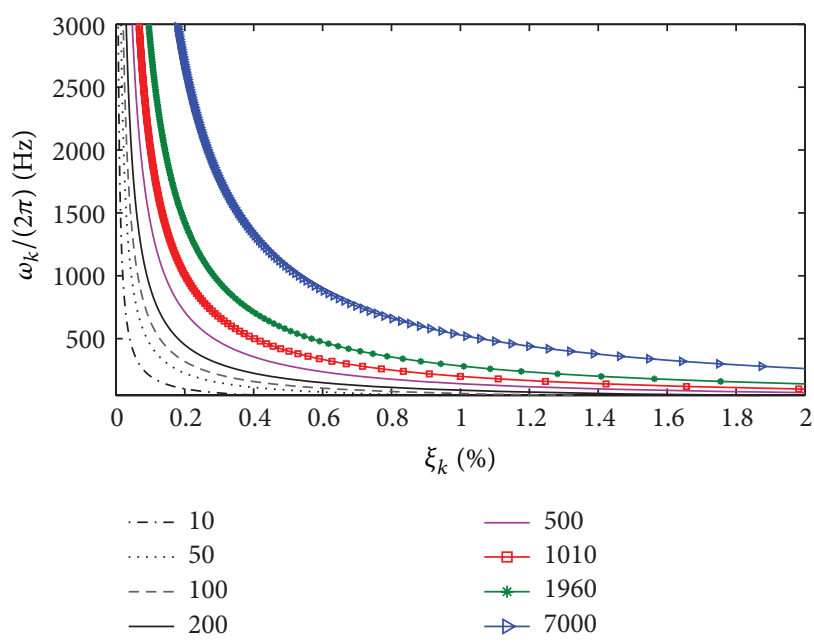

FIGURE 19: Relationship between the values of $\omega_{k}$ and $\xi_{k}$ which allows having a value of Perf $\left|T_{z w}\right|_{\max }$ equal to $5 \mathrm{~dB}$ for different values of $\psi_{k}^{2} \bar{\gamma}\left(\right.$ in $\left.\mathrm{rad}^{2} / \mathrm{s}^{2}\right)$.

values increase with increasing $\psi_{k}^{2} \bar{\gamma}$ (Figures 17 and 18). This analysis brings to light the maximum performance feasible for a given problem and provides significant information on the efficacy of this kind of control technique in accordance with the variation of the modal parameters of the mode to control. Certain limits to its applicability are plainly evident indeed. For example, if an attenuation of at least $5 \mathrm{~dB}$ is required, Figures 17 and 18 show that, in shifting from the lowest to the highest value of $\psi_{k}^{2} \bar{\gamma}$ (see Table 4 ), the maximum frequency at which this threshold can be respected decreases with the increase of the mechanical damping. Figure 19 shows the relation between $\omega_{k}$ and $\xi_{k}$ for different values of $\psi_{k}^{2} \bar{\gamma}$, when an attenuation (in terms of Perf $\left|T_{z w}\right|_{\max }$ ) of $5 \mathrm{~dB}$ is required. This last figure clearly describes an asymptotic behaviour and shows the limits of applicability of the shunt damping.

Figures 14 and 15 illustrate the dependency of the performance on $\omega_{k}, \xi_{k}$, and $\psi_{k}^{2} \bar{\gamma}$. As previously reckoned, $\omega_{k}$ and $\xi_{k}$ depend exclusively on the nature of the problem and cannot be modified to yield better performances; moreover, their values limit the applicability of control strategies based on shunted piezo-actuators. The third parameter (i.e., $\psi_{k}^{2} \bar{\gamma}$ ) depends instead on the type of piezo-actuator used and on its position on the structure: it can therefore be modified. The following section discusses the influence of $\psi_{k}^{2} \bar{\gamma}$ on the performance and how it is possible to act on this parameter in order to achieve the best results.

4.4. Influence of $\psi_{k}^{2} \bar{\gamma}$ on the Controller Performance. Section 4.3 has evidenced how the performance of the shunt control depends on the problem parameters $\omega_{k}, \xi_{k}$, and $\psi_{k}^{2} \bar{\gamma} \cdot \psi_{k}^{2} \bar{\gamma}$ is the only parameter which can be modified in order to maximise the vibration reduction for a given problem. The effect of $\psi_{k}^{2} \bar{\gamma}$ on the performance can be inferred from Figures 14 and 15: performance improves with the increase of $\psi_{k}^{2} \bar{\gamma}$. This effect is made the more evident by 


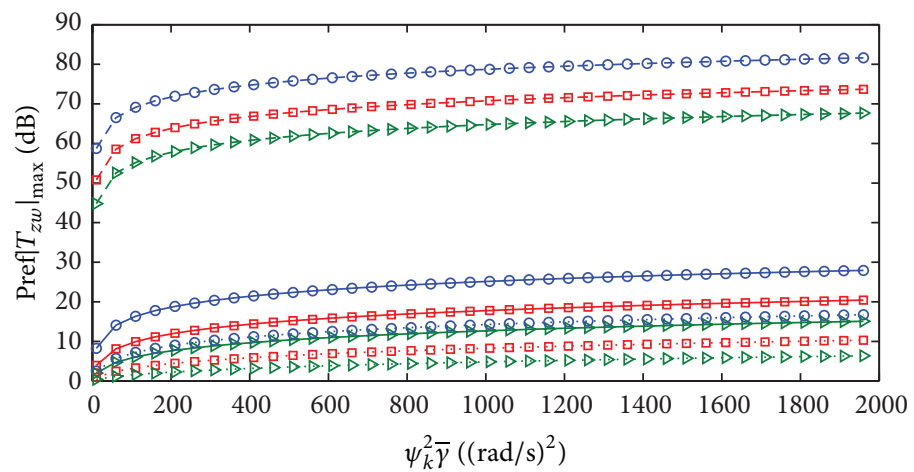

Figure 20: Perf|T $\left.T_{z w}\right|_{\max }$ as function of $\psi_{k}^{2} \bar{\gamma}$. The dashed lines refer to $\xi_{k \text { low }}$, the solid lines refer to $\xi_{k \text { medium }}$, and the dotted lines refer to $\xi_{k \text { high }}$. The circles identify $\omega_{k_{\text {low }}}$, the squares $\omega_{k_{\text {medium }}}$, and the triangles $\omega_{k_{\text {high }}}$ (see Table 4$)$.

Figure 20: illustrating the performance as function of $\psi_{k}^{2} \bar{\gamma}$ for different values of damping and natural frequency of the mechanical mode considered. A monotonic increasing trend of the performance in accordance with the increase of $\psi_{k}^{2} \bar{\gamma}$ can be recognized in Figure 20: hence, the increase of $\psi_{k}^{2} \bar{\gamma}$ is desirable. This parameter is composed of two terms: $\psi_{k}^{2}$, which depends on the position of the piezo-actuator on the structure, and $\bar{\gamma}$, which depends on electrical, mechanical, and geometrical features of the piezo-actuator and the structure (see Tables 1 and 2). This means that proper choice of material and geometry of the actuator influence the possibility of increasing the value of $\bar{\gamma}$ (e.g., $\bar{\gamma}$ increases with increasing $E_{p}$ and $\nu_{p}$ ) and the amount of that increment. If instead $\psi_{k}^{2}$ is considered, the position of the actuator should be optimised. Generally speaking, the best position is the area of the structure in which the mode considered features maximum curvature, as can be derived from the formulas in Table 1. Furthermore, some methods are available in literature (e.g., [15]) to properly place the piezo-actuator on the structure, leading to the best value of $\psi_{k}^{2}$ for the problem under analysis.

It is noteworthy that the performance as function of $\psi_{k}^{2} \bar{\gamma}$ displays an asymptotic trend (Figure 20). Therefore, in certain practical cases the value of $\psi_{k}^{2} \bar{\gamma}$ cannot be maximised to achieve the desired performance. Moreover, efforts to increase $\psi_{k}^{2} \bar{\gamma}$ may be too high in terms of cost, with respect to the improvement in performance. In fact, the slope of the curves of Figure 20 decreases significantly for values of $\psi_{k}^{2} \bar{\gamma}$ higher than $400 \mathrm{rad}^{2} / \mathrm{s}^{2}$ (e.g., the rate of increase is just about $0.003 \mathrm{~dB} /(\mathrm{rad} / \mathrm{s})^{2}$ between 1000 and $\left.2000 \mathrm{rad}^{2} / \mathrm{s}^{2}\right)$.

A further significant aspect brought to light through this general approach and related to the dependency of the performance on $\bar{\gamma}$ (Figure 20 ) is the possibility to vary the values of $R$ and $L$ obtained by the optimisation procedure maintaining the same level of performance. The optimum value of $L$ is often very high and this makes it impossible to assemble the network with passive electrical components (e.g., [3]). Some problems can be encountered also for the value of $R$. In fact, the required optimal resistance value is very low (e.g., one or two tens of Ohm) in some cases and there are situations where it is not possible to have such a low resistance since the sole resistance of cables and piezo-patch [20] are higher than the optimum one.

Based on (31), (32), and (45) the optimal resistance and inductance values assume the following expressions:

$$
\begin{aligned}
L_{\mathrm{opt}} & =\frac{1}{C_{p} \omega_{p}^{2}}=\frac{1}{C_{p}\left(\omega_{k}^{2}+\psi_{k}^{2} \bar{\gamma}\right)}, \\
R_{\mathrm{opt}} & =\frac{2 \xi_{p}}{C_{p} \sqrt{\omega_{k}^{2}+\psi_{k}^{2} \bar{\gamma}}} .
\end{aligned}
$$

These relations show that the optimal values of resistance and inductance can be changed by varying the capacitance of the piezo-actuator $C_{p}$, setting the performances of the controller (i.e., $\psi_{k}^{2} \bar{\gamma}=$ costant). In fact, an important aspect reckoned by this general approach concerns the fact that the desired performance can be maintained, even varying the piezo-capacitance, by acting on other electrical, geometrical, and mechanical parameters of the piezo-actuator. In fact, the value of $\psi_{k}^{2} \bar{\gamma}$ must remain constant in order to maintain the desired performance. A change of the $C_{p}$ value leads to a change of $\psi_{k}^{2} \bar{\gamma}$. However, Table 1 shows that this change can be compensated by a change of other piezo-parameters (e.g., the piezo-actuator thickness) allowing maintaining the desired performance level. Therefore, $C_{p}$ can be changed, but also the other parameters of $\bar{\gamma}$ (Tables 1 and 2) can be modified, so to compensate the change of $C_{p}$. This leads to a change of $L$ and $R$ values, without varying the attenuation performance. Usually, the inductance value needs to be reduced, thus a higher value of capacitance is required. Conversely, the capacitance should be reduced to increase $R_{\text {opt }}$. The effect of $C_{p}$ in the two cases is opposite.

Thus, the problem of high inductance values can be partially solved by increasing $C_{p}$ and changing the other parameters accordingly, so to upkeep the performance level. Equation (59) shows that when $C_{p}$ is doubled, the resulting $L_{\text {opt }}$ decreases by a factor of 2 if the value of $\psi_{k}^{2} \bar{\gamma}$ is maintained constant. Instead, if the value of $\psi_{k}^{2} \bar{\gamma}$ is not maintained, it decreases by a factor of 2 (Table 1), but this does not influence the value of $L_{\text {opt }}$ because the term $\omega_{k}^{2}+\psi_{k}^{2} \bar{\gamma}$ (59) depends nearly entirely on $\omega_{k}^{2}$ in the more commonplace applications. 
Furthermore, if the original value of $\psi_{k}^{2} \bar{\gamma}$ is higher than about $800 \mathrm{rad}^{2} / \mathrm{s}^{2}$, decreasing it by a factor of 2 does not change the performance significantly (Figure 20).

As for the problem of low values of $R_{\text {opt }},(60)$ shows that a decrease of $C_{p}$ allows increasing $R_{\text {opt }}$ and at the same time improves the attenuation performance (Table 1). Furthermore, the consequent increase of $\psi_{k}^{2} \bar{\gamma}$ (Table 1) additionally increases the optimal value of $\xi_{p}$ (e.g., (53)) and of $R_{\text {opt }}(60)$. Hence, a hypothetical decrease of $C_{p}$ by a factor of 2 allows increasing $R_{\text {opt }}$ by a factor of 4 (it shall be remarked that the term $\omega_{k}^{2}+\psi_{k}^{2} \bar{\gamma}$ of (60) depends nearly entirely on $\omega_{k}^{2}$ in commonplace applications). The possibility to increase $R_{\mathrm{opt}}$ is of particular importance, especially when its initial value is small. Indeed, two problems can arise from low values of $R_{\text {opt }}$. First, a small change of the shunt resistance (e.g., due to thermal shifts [31]) can result in a high percentage change of $\xi_{p}$ with respect to the optimal value, and thus resulting in a corresponding significant decrease of the attenuation performance. Furthermore, the resistance of the cables and of the piezo-patch [20] can be even higher than the optimum resistance value.

This formulation remarkably can evidence the limits of applicability of such a kind of control strategy, as well as the means to improve performances when possible. Moreover, the asymptotic behaviour illustrated by Figure 20 shows that the optimisation of all parameters wholesale is not in fact useful in some cases. Finally, it shall be remarked that this approach and conclusion are general and hence valid for each and all of the optimisation methods, but above all they are valid for all structures in diverse configurations (see Table 2).

\section{Robustness Analysis}

The performance of the tuning methodologies proposed in Section 3 has been discussed in Section 4. All the methods featured similar performances in terms of indexes $\operatorname{Perf}\left|T_{z w}\right|_{\max }$ and Perf $\left|T_{z w}\right|_{\omega_{k}}$, with the exception of optimisation 3 which proved to be more effective in case of monoharmonic excitation at resonance (i.e., index $\operatorname{Perf}\left|T_{z w}\right|_{\omega_{k}}$ ). All these results can be considered valid, as long as all the mechanical and electrical parameters are known without uncertainties, thus allowing to achieve perfect tuning. This condition is rather unrealistic and hardly ever found in real applications. In fact, the modal parameters of the structure to control can easily change (e.g., a shift of $\omega_{k}$ due to a temperature variation). Furthermore, the uncertainty affecting the estimates of the electrical parameters such as $R, L$, and $C_{p}$ is often not negligible. These aspects altogether lead in most cases to the mistuning of the control system (even when starting from a condition of perfect tuning), and therefore the performance results are different from expected. In spite of its evident importance, a performance analysis of the control strategies in case of mistuning has never been carried out and presented in literature. The authors of this paper have thus decided to test the control strategies proposed also in mistuned conditions.

This section discusses a robustness analysis undertaken with the aim of investigating the behaviour of some of the tuning methodologies presented so far, in cases in which uncertainty affects the estimates of the modal, electrical, and mechanical parameters. The analysis does not take into account all of the six methods described in Section 3, but only three of those have been selected. Figure 13 illustrates that the six methods can in fact be divided into three groups, relying on their optimal electrical damping $\xi_{p}$. Within each of the three groups, the performances of the different methods have resulted to be similar, and for this reason the authors have selected from each group only one method on which to conduct the robustness analysis: optimisation 1 for the first group, optimisation 2-und for the second, and optimisation 3 for the third.

As already outlined, uncertainties can affect the estimates of the parameters involved in the problem and biases on $\omega_{k}, \xi_{k}, \psi_{k}^{2} \bar{\gamma}, R, L$, and $C_{p}$ should be taken into account in the robustness analysis. Since the bias on each of these parameters translates into a mistuning of the control system, the authors have converted all of these uncertainties into biases of the optimal value of the electrical frequency $\omega_{p}$ and damping $\xi_{p}$, for the robustness analysis. The bias of these quantities will be expressed as a percentage of the optimal value herein.

The results of the robustness analysis are presented in the following two subsections: a comparison between the three methods chosen, with varying the biases on $\omega_{p}$ and $\xi_{p}$, is provided in Section 5.1, while Section 5.2 delves into a more detailed comparison between two particular methods.

5.1. Robustness Comparison. The behaviour of the three tuning methodologies in terms of the indexes of (58) in presence of biases on $\omega_{p}$ and $\xi_{p}$ has been investigated for all the 27 cases of Table 5. Simulations were performed considering biases up to $100 \%$ on the electrical damping and of $2 \%$ on the electrical frequency for each case: $0<\xi_{p}<2 \xi_{p_{\text {ott }}}$ and $0.98 \omega_{p_{\text {ott }}}<$ $\omega_{p}<1.02 \omega_{p_{\text {ott }}}$. Thus, the biases $\left(\varepsilon_{\omega_{p}}\right.$ and $\left.\varepsilon_{\xi_{p}}\right)$ are expressed in terms of percentage of the optimal values. The values for $\xi_{p_{\text {ott }}}$ are often quite small (i.e., $0.0002<\xi_{p}<0.28$ for the working domain described in Section 4.1) and therefore a high percentage error is considered in the simulations. Conversely, the values of $\omega_{p}$ are high and therefore a $2 \%$ error on the optimal value can be considered high.

Figures 21 and 22 illustrate a small number of cases that have been selected as representative of the behaviour of the methods in response to the variation of the problem parameters of Table 3. Both figures show which method (among the three selected) achieves the best performance in presence of biases on the tuning of the shunt impedance.

As for Figure 21, optimisation 1 features the best behaviour in case of mistuning: it provides the best attenuation in terms of Perf $\left|T_{z w}\right|_{\max }$ for a wide range of biases, on both electrical damping and frequency. Comparing Figures 21(b) and 21(a) evidences that the area in which optimisation 1 works better than the other two increases with increasing of $\omega_{k}$; the area is instead almost unchanged with increasing the damping value (i.e., Figure 21(a) compared to Figure 21(d)). Instead, if $\psi_{k}^{2} \bar{\gamma}$ increases, the areas of optimisations 2 -und and 3 tend to increase (i.e., Figure 21(a) compared to Figure 21(c)). 


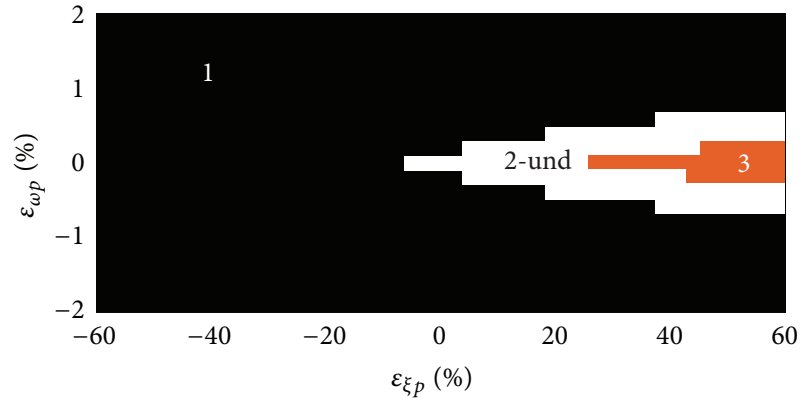

(a)

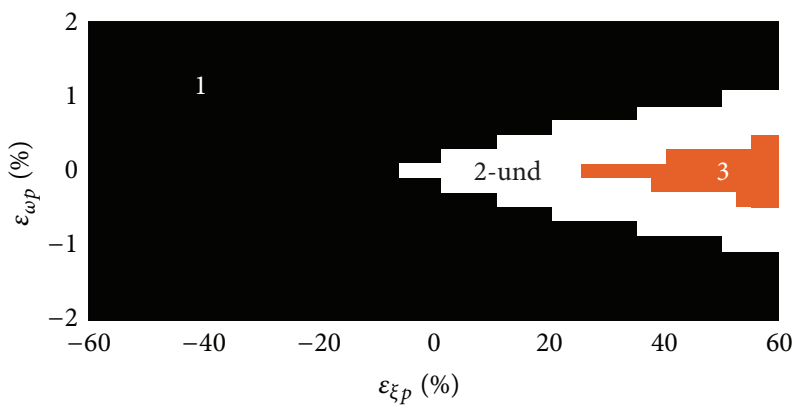

(c)

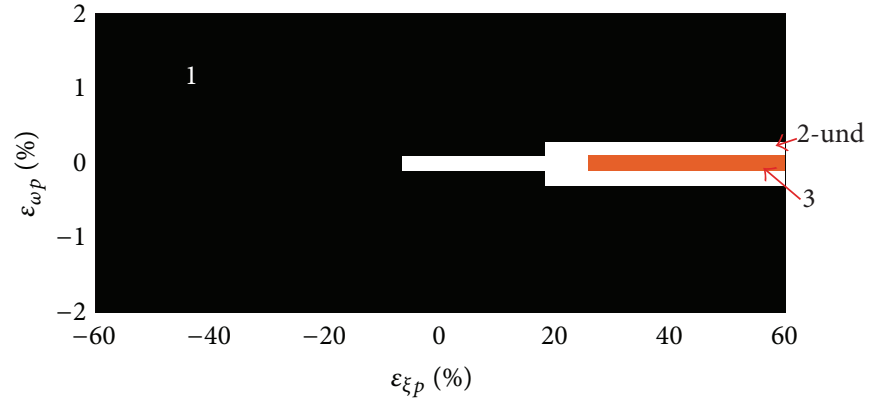

(b)

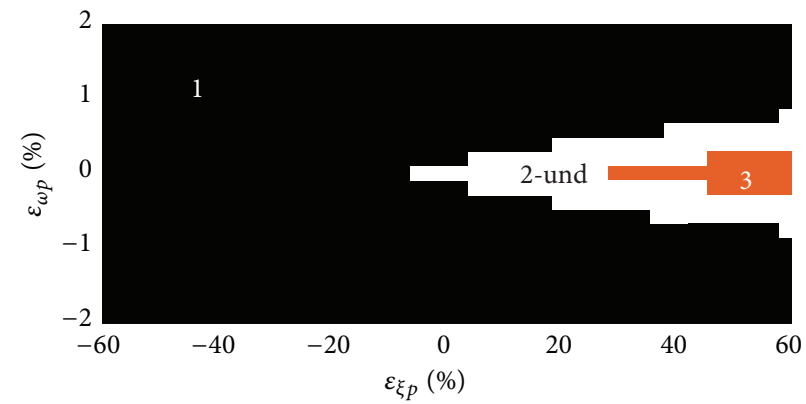

(d)

FIGURE 21: Maps showing the method featuring the best performance in terms of Perf $\left|T_{z w}\right|_{\max }$ for a given bias on $\xi_{p}$ and $\omega_{p}$. (a) Case 13 (refer to Table 5); (b) case 22; (c) case 16; and (d) case 14 . The bias values on $\xi_{p}$ were limited to $\pm 60 \%$.

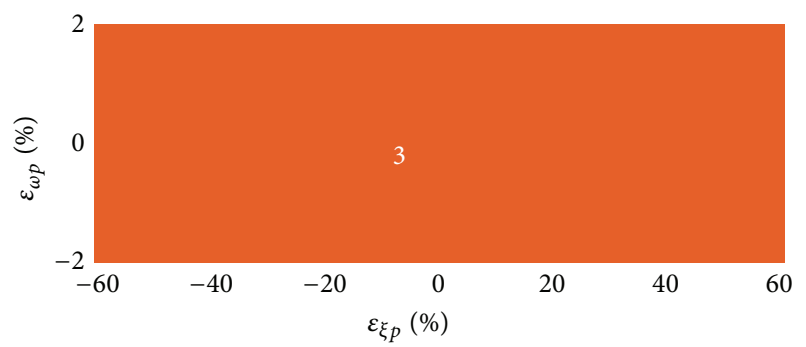

(a)

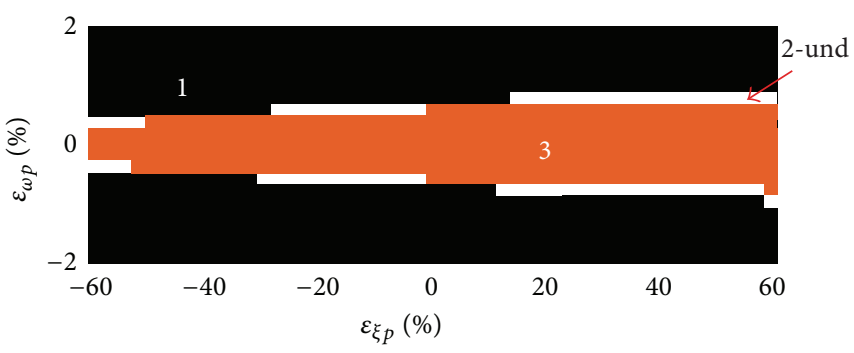

(c)

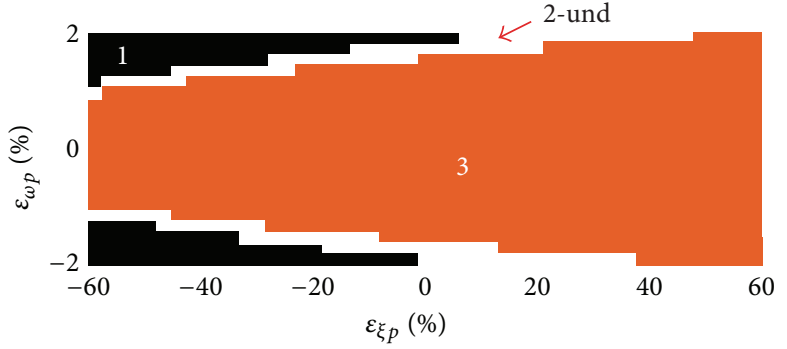

(b)

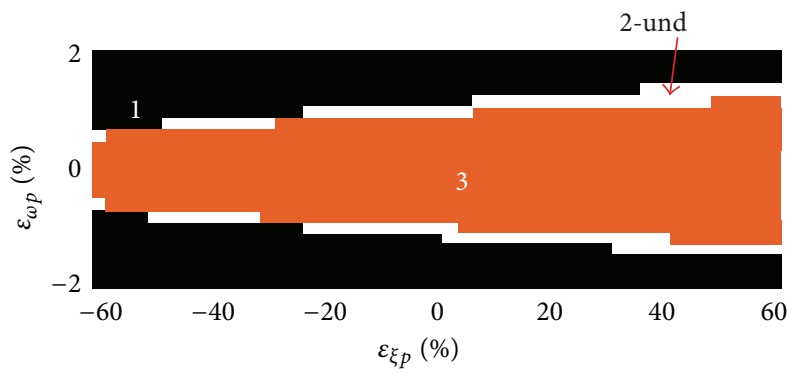

(d)

FIGURE 22: Maps showing the method featuring the best performance in terms of Perf|T $\left.T_{z w}\right|_{\omega_{k}}$ for a given bias on $\xi_{p}$ and $\omega_{p}$. (a) Case 13 (refer to Table 5); (b) case 14 ; (c) case 23 ; and (d) case 26 . The bias values on $\xi_{p}$ were limited to $\pm 60 \%$.

Considering the performance in case of monoharmonic excitation at resonance, Figure 22 shows that optimisation 3 is the best choice, even in conditions of mistuning. In fact, optimisation 1 is preferable only in case of high biases on $\omega_{p}$ when the structural damping or the natural frequency is increased (i.e., Figures 22(b) and 22(c)). For high values of $\psi_{k}^{2} \bar{\gamma}$ optimization 3 is more effective, also with high $\omega_{k}$ and $\xi_{k}$ (i.e., Figure 22(d) compared to Figure 22(c)).

These analyses show that optimisation 1 is the best choice in the case of a wide band excitation around $\omega_{k}$ 


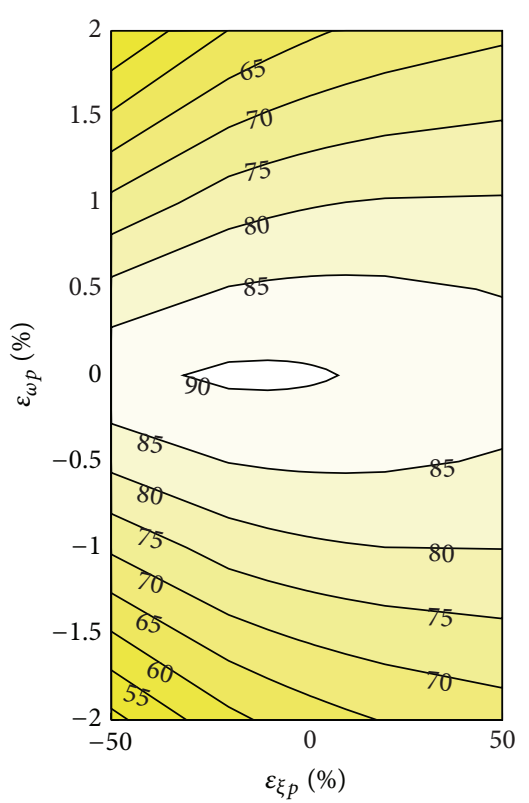

(a)

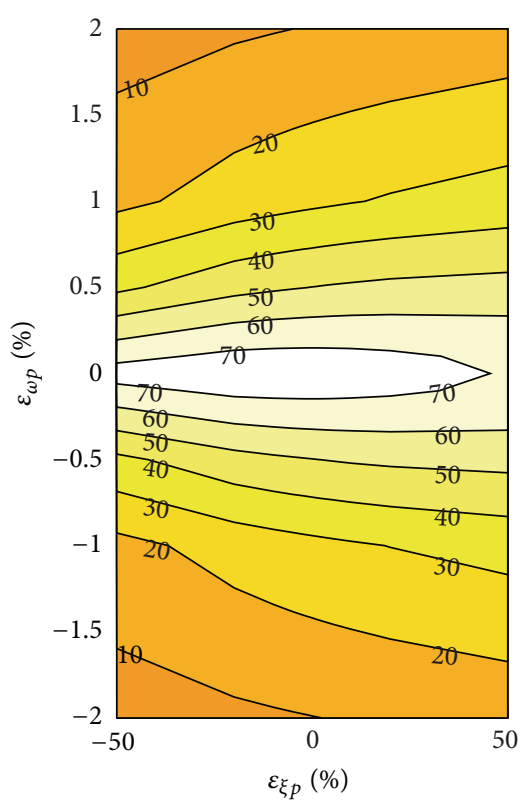

(b)

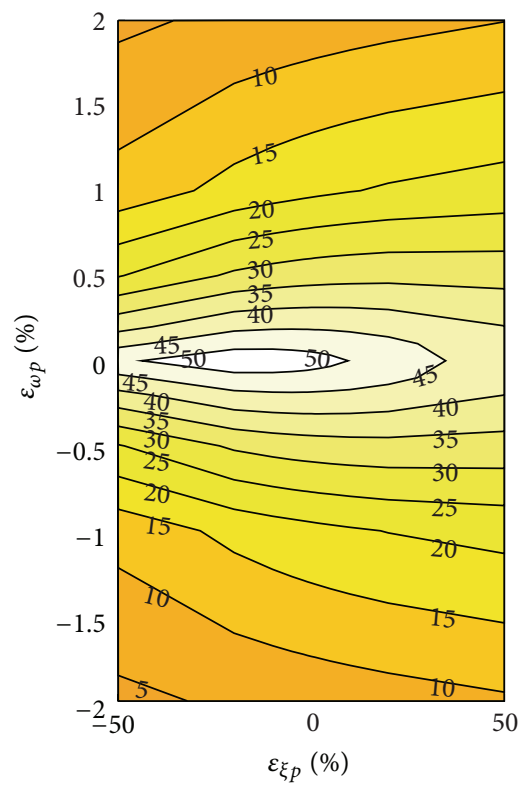

(c)

FIgURE 23: $\eta_{1}$ for case 17 (a), case 23 (b), and case 27 (c).

(i.e., Perf $\left|T_{z w}\right|_{\max }$ ), while optimisation 3 should be employed in case of monoharmonic excitation at resonance (i.e., $\left.\operatorname{Perf}\left|T_{z w}\right|_{\omega_{k}}\right)$. Since this latter case is quite uncommon in practice, the authors have decided to carry out all the further analyses and experimental tests considering wide band excitation. Optimisations 1 and 2-und are compared with further details in the following subsection.

5.2. Performances in Mistuned Conditions. The behaviour of optimisation 1 and 2-und in mistuned conditions is described in Section 5.1: optimisation 1 achieves better results than 2und for negative errors on $\xi_{p}$ and in case of positive errors on $\xi_{p}$ together with a high bias of $\omega_{p}$ (Figure 21). This section examines and quantifies the difference between the two methods so to evidence the performance improvement achievable with either of them.

The index used to quantify the difference in terms of performance at the maximum value of $\left|T_{z w}\right|$ is

$$
\begin{aligned}
& \mathcal{E}_{\text {perf }}[\%] \\
& =\frac{\left(\left|T_{z w}\right|_{\text {max }_{\mathrm{nc}}}-\left|T_{z w}\right|_{\text {max }_{\mathrm{ott1}}}\right)-\left(\left|T_{z w}\right|_{\text {max }_{\mathrm{nc}}}-\left|T_{z w}\right|_{\text {max }_{\mathrm{ott} 2-\mathrm{und}}}\right)}{\left|T_{z w}\right|_{\text {max }_{\mathrm{nc}}}-\left|T_{z w}\right|_{\text {max }_{\mathrm{ottl}}}} \\
& \cdot 100,
\end{aligned}
$$

where $\left|T_{z w}\right|_{\max _{\text {ott }}}$ is the maximum value of the dynamic amplification modulus achieved using optimisation 1, $\left|T_{z w}\right|_{\text {max }_{\text {ott2-und }}}$ represents the maximum value of the dynamic amplification modulus achieved using optimisation 2-und, and $\left|T_{z w}\right|_{\text {max }_{\mathrm{nc}}}$ is the maximum value with no control.

$\varepsilon_{\text {perf }}$ brings to light the differences in terms of performance between the two methods, normalised on the vibration attenuation achievable in a specific mistuned condition by using optimisation 1 . Indeed, a given difference between the methods can be considered high if in that mistuned condition the vibration attenuation is low, whereas the same difference becomes negligible if the vibration attenuation is high. Equation (61) provides unambiguous information about the performance of the two methods in all of the mistuned condition.

Figure 23 contains a representative selection of three of the 27 cases listed in Table 5, to illustrate the behaviour of the two methods. Figures 23(a), 23(b), and 23(c) show the performance of optimisation 1 in terms of vibration reduction percentage, compared to the uncontrolled case for different mistuned conditions:

$$
\eta_{1}=\frac{\left|T_{z w}\right|_{\max _{\mathrm{nc}}}-\left|T_{z w}\right|_{\max _{\mathrm{ott1}}}}{\left|T_{z w}\right|_{\max _{\mathrm{nc}}}} 100 .
$$

The cases selected and presented in Figure 23 are numbers 17, 23, and 27 of Table 5. The control is highly effective in Figure 23(a) because $\omega_{k}, \xi_{k}$, and $\psi_{k}^{2} \bar{\gamma}$ have favourable values for a high vibration reduction (see Sections 4.3 and 4.4). Figure 23(a) shows that in this case $\eta_{1}$ is very high also with high biases on the electrical damping and the electrical frequency. In the second and third cases (Figures 23(b) and $23(\mathrm{c})), \omega_{k}$ and $\xi_{k}$ are increased and the maximum performance drops, as well as the behaviour in mistuned conditions.

The differences between optimisations 1 and 2-und are less relevant in cases in which the performances of the shunt control are high (case 17, Figure 24(a)) than in the cases in which the maximum possible vibration reduction decreases (cases 23 and 27, Figures 24(b) and 24(c)). Optimisation 1 can improve the vibration reduction in case of mistuned conditions more than optimisation 2-und, in cases where 




(a)

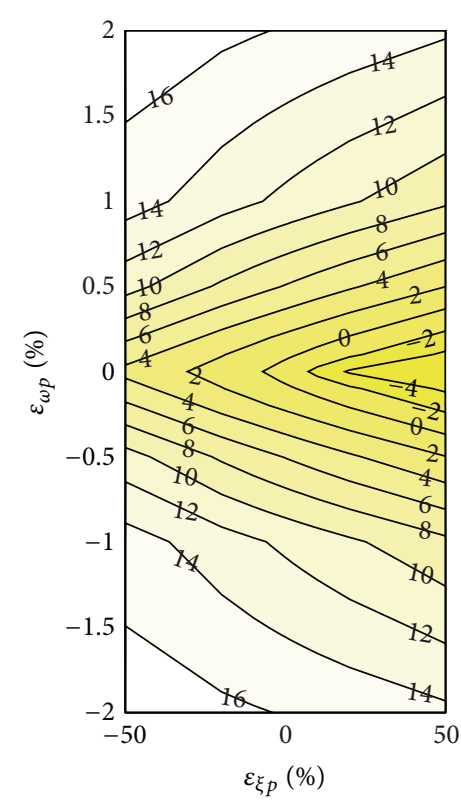

(b)

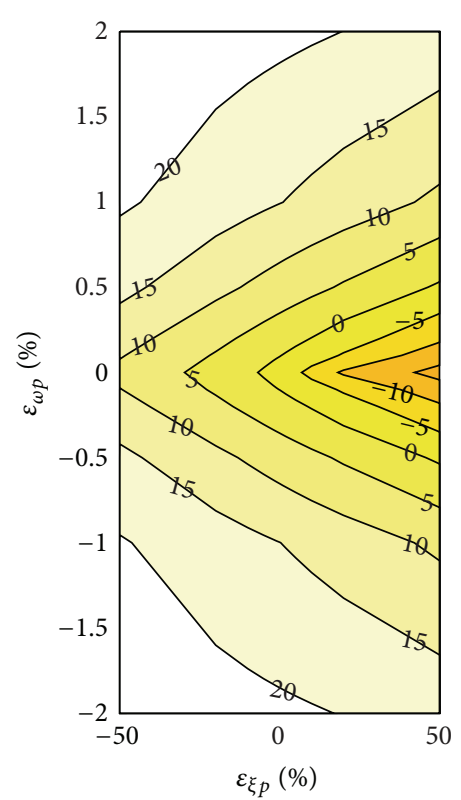

(c)

FIgURE 24: $\varepsilon_{\text {perf }}$ for case 17 (a), case 23 (b), and case 27 (c).

the mode is not easily controllable (i.e., cases 23 and 27). In fact, optimisation 2-und leads to improvements of $\varepsilon_{\text {perf }}$ only in a narrow area of biases.

The analyses presented in Sections 4.3 and 4.4 show that optimisation 1 allows reaching performances as good as with the other tuning methodologies, when there are no uncertainties on the parameters. Moreover, it is more robust than the other methods in case of mistuning, thanks to higher damping values (Figure 13).

Yet, the paramount observation to be made concerns the shape of plots in Figures 23(a), 23(b), and 23(c). These plots are stretched out along the horizontal axis and the level curves tend to open, proceeding from underestimations of $\xi_{p}$ towards overestimations. This indicates that overestimations of $\xi_{p}$ are less problematic than underestimations with a mistuning on $\omega_{p}$, whatever the structure considered. It is noteworthy that when the shunt control works in the right part of the plots (i.e., overestimation of $\xi_{p}$ ), the robustness always increases. Therefore, it is recommendable to set the value of $\xi_{p}$ higher than about $20-25 \%$ of the optimum value. This provides for a significant increase of the shunt control robustness.

Finally, Figures 25 and 26 show the value of $\eta_{1}$ for a bias on $\omega_{p}$ of $+2 \%$ of the optimal value and bias values on $\xi_{p}$ in the range of $\pm 20 \%$ of the optimal value, for method 1 and for some of the cases of Table 5 (cases neglected are those in which the bias values used are too high thus causing extremely poor performance or cases with a very high performance which are not disturbed by bias values and would thus require higher biases to show any significant drop in performance). Furthermore, the same plots show the trend of $\eta_{1}$ when the initial value of $\xi_{p}$ is purposefully increased or decreased by $25 \%$ (i.e., the mistuned value of $\xi_{p}$ is in the ranges $5 \div 45 \%$ and $-45 \div-5 \%$ of the optimal value of $\xi_{p}$ resp.). The benefits
TABLE 6: Test setup nominal data.

\begin{tabular}{lc}
\hline Parameter & Value \\
\hline Plate length & $600 \mathrm{~mm}$ \\
Plate width & $400 \mathrm{~mm}$ \\
Plate thickness $(h)$ & $8 \mathrm{~mm}$ \\
Density of the plate material & $2700 \mathrm{~kg} / \mathrm{m}^{3}$ \\
Poisson's modulus of the plate material $(\nu)$ & 0.3 \\
Young's modulus of the plate material $(E)$ & $72 \mathrm{GPa}$ \\
Length of the bender $\left(l_{p}\right)$ & $46 \mathrm{~mm}$ \\
Width of the bender $\left(w_{p}\right)$ & $33 \mathrm{~mm}$ \\
Thickness of the bender $\left(t_{p}\right)$ & $0.13 \mathrm{~mm}$ \\
Young's modulus of the bender $\left(E_{p}\right)$ & $65 \mathrm{Gpa}$ \\
Piezoelectric strain constant $\left(d_{31}\right)$ & $-179 \cdot 10^{-12} \mathrm{~m} / \mathrm{V}$ \\
Capacitance of the bender $\left(C_{p}\right)$ & $0.1 \mu \mathrm{F}$ \\
Poisson's modulus of the bender $\left(\nu_{p}\right)$ & 0.34 \\
\hline
\end{tabular}

in terms of robustness from using an overestimated $\xi_{p}$ value are thus evident.

The following section will validate the results shown so far.

\section{Experimental Tests on a Plate Structure}

This section describes the experimental tests carried out to validate the results unto here discussed in the previous sections. The structure used in the tests is an aluminium plate, with the shunt piezo-bender bonded close to its centre. A 2D structure was used, since this constitutes a more complex and thus interesting test case than 1D structures often used in other works. The technical data of the test setup are provided in Table 6. 


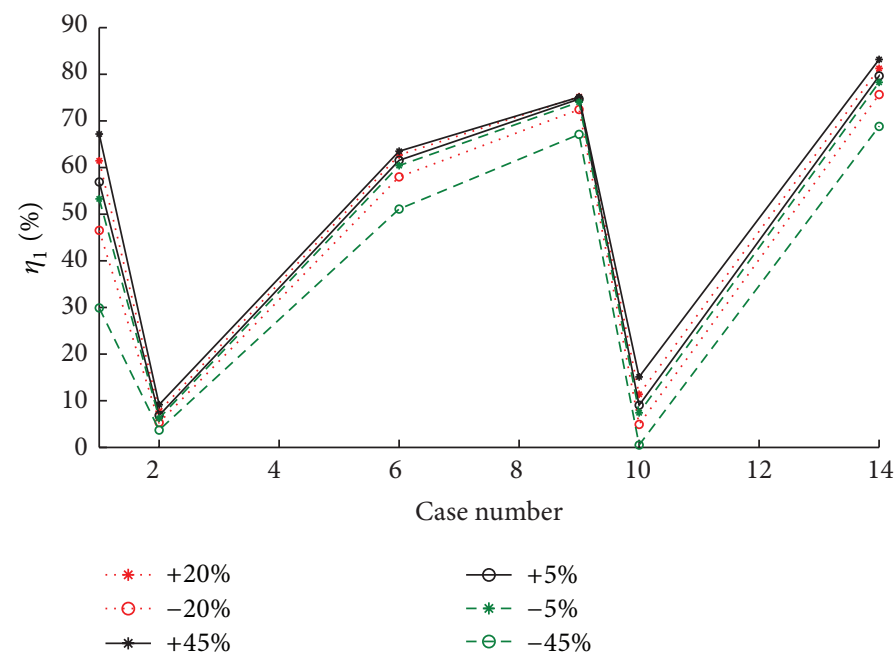

Figure 25: $\eta_{1}$ for different cases of Table 5 and values of bias on $\xi_{p}$ (bias on $\omega_{p}$ equal to $+2 \%$ ).

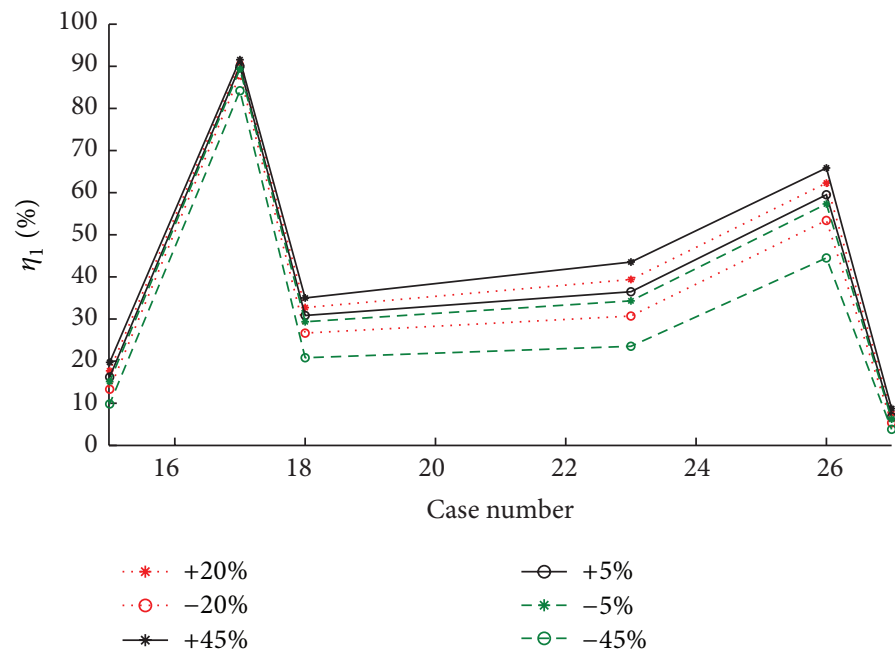

Figure 26: $\eta_{1}$ for different cases of Table 5 and values of bias on $\xi_{p}$ (bias on $\omega_{p}$ equal to $+2 \%$ ).

The tests were carried out in two different ways: the first employed a synthetic impedance based on operational amplifiers [16] to simulate the inductor, the second simulated the whole shunt impedance by means of a high-speed Field Programmable Gate Array (FPGA) device (in this latter case a colocated piezo-patch was used to provide the input voltage to the simulated shunt impedance; this second patch was nominally equal to that used to provide the control action). The two techniques led to similar results. The disturbance was provided by means of a dynamometric impact hammer, while the response of the structure was collected by means of an accelerometer.

The modes of the structure used in the tests were the third and the fourth (Table 7), identified by means of an experimental modal analysis.

Several tests were performed on the plate in order to validate the analytical and numerical results shown in the previous sections. First of all, we validated the analytical model of the controlled electromechanical structure explained in
TABLE 7: Identified modal data.

\begin{tabular}{lccc}
\hline Mode number & $\begin{array}{c}\omega_{k} \\
{[\mathrm{rad} / \mathrm{s}]}\end{array}$ & $\begin{array}{c}\xi_{k} \\
{[\%]}\end{array}$ & $\begin{array}{c}\psi_{k}^{2} \bar{\gamma} \\
{\left[(\mathrm{rad} / \mathrm{s})^{2}\right]}\end{array}$ \\
\hline Mode 3 & $530.67 \cdot 2 \pi$ & 0.22 & 95.7 \\
Mode 4 & $662.10 \cdot 2 \pi$ & 0.15 & 147.4 \\
\hline
\end{tabular}

Section 2. Since the response of the test structure was measured using accelerometers, we compared the experimental results and the numerical simulations in terms of $T_{z w a c c}$. $T_{z w a c c}$ is the transfer function between the disturbance $W$ and the structural response in terms of acceleration (i.e., $\ddot{z}$ ) and it can be easily derived analytically relying on (34):

$$
\begin{aligned}
& T_{z w a c c}=T_{z w} s^{2}=s^{2} \Phi_{k}\left(x_{M}\right) \Phi_{k}\left(x_{F}\right) \\
& \cdot \frac{\left(s^{2}+2 \xi_{p} \omega_{p} s+\omega_{p}^{2}\right)}{\left(s^{2}+2 \xi_{p} \omega_{p} s+\omega_{p}^{2}\right)\left(s^{2}+2 \xi_{k} \omega_{k} s+\omega_{k}^{2}\right)+\bar{\gamma} \psi_{k}^{2} s\left(s+2 \xi_{p} \omega_{p}\right)} .
\end{aligned}
$$


TABLE 8: Test cases for Mode 3.

\begin{tabular}{|c|c|c|c|c|c|c|c|c|c|}
\hline & \multicolumn{6}{|c|}{ Mode 3} & \multirow{2}{*}{\multicolumn{2}{|c|}{$D$}} \\
\hline & & \multicolumn{2}{|c|}{$A$} & \multicolumn{2}{|r|}{$B$} & \multicolumn{2}{|c|}{$C$} & & \\
\hline & & $\varepsilon_{\xi_{p}}=-40 \%$ & $\varepsilon_{\omega_{p}}=0 \%$ & $\varepsilon_{\xi_{p}}=-40 \%$ & $\varepsilon_{\omega_{p}}=-0.175 \%$ & $\varepsilon_{\xi_{p}}=-90 \%$ & $\varepsilon_{\omega_{p}}=0 \%$ & $\varepsilon_{\xi_{p}}=-90 \%$ & $\varepsilon_{\omega_{p}}=0.2 \%$ \\
\hline \multirow{2}{*}{$\varepsilon_{\text {perf }}$} & Experimental & 12 & & & 23.0 & \multicolumn{2}{|c|}{29.0} & \multicolumn{2}{|c|}{35.8} \\
\hline & Numerical & 12 & & & 21.7 & 28 & & \multicolumn{2}{|c|}{32.4} \\
\hline
\end{tabular}

TABle 9: Test cases for Mode 4.



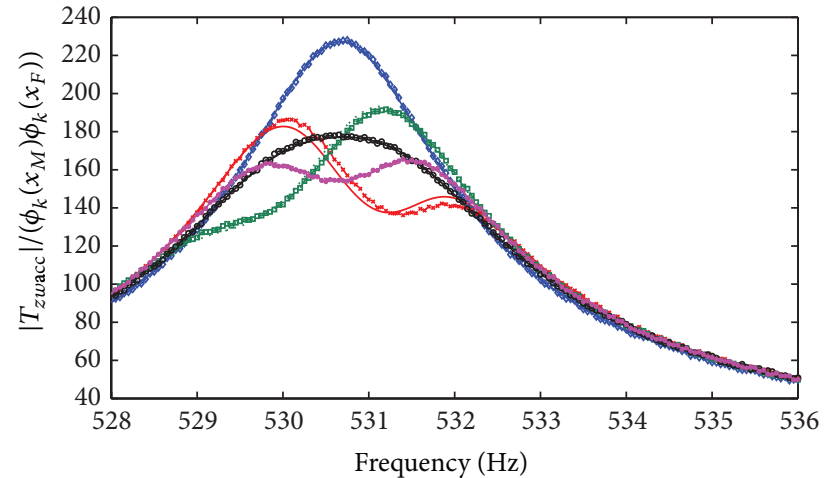

$$
\begin{aligned}
& -- \text { Short circuit-num } \\
& -\omega_{p}=3327.6 \mathrm{rad} / \mathrm{s}, \xi_{p}=0.217 \% \text {-num } \\
& \cdots \cdots \omega_{p}=3337.6 \mathrm{rad} / \mathrm{s}, \xi_{p}=0.166 \% \text {-num } \\
& --\omega_{p}=3334.3 \mathrm{rad} / \mathrm{s}, \xi_{p}=0.347 \% \text {-num } \\
& -\omega_{p}=3334.1 \mathrm{rad} / \mathrm{s}(\mathrm{eq} .(45)), \xi_{p}=0.205 \% \text { (eq. (53))-num } \\
& -\diamond-\text { Short circuit-exp } \\
& -*-\omega_{p}=3327.6 \mathrm{rad} / \mathrm{s}, \xi_{p}=0.217 \% \text {-exp } \\
& - \text { - } \omega_{p}=3337.6 \mathrm{rad} / \mathrm{s}, \xi_{p}=0.166 \% \text {-exp } \\
& -\bullet-\omega_{p}=3334.3 \mathrm{rad} / \mathrm{s}, \xi_{p}=0.347 \% \text {-exp } \\
& -*-\omega_{p}=3334.1 \mathrm{rad} / \mathrm{s}(\text { eq. }(45)), \xi_{p}=0.205 \% \text { (eq. (53))-exp }
\end{aligned}
$$

FIGURE 27: Numerical and experimental trend of $\left|T_{z w a c c}\right|$ (normalised over $\left.\Phi_{k}\left(x_{M}\right) \Phi_{k}\left(x_{F}\right)\right)$ for the mode at about $530 \mathrm{~Hz}$ (Table 7) and fixed values of $\omega_{p}$ and $\xi_{p}$.

The tests were carried out imposing different values of the electrical eigenfrequency $\omega_{p}$ and the electrical damping $\xi_{p}$ and looking at the response of the modes in Table 7. Figures 27 and 28 show the comparison between experimental and analytical FRFs for the uncontrolled case (i.e., short-circuit), for several $\omega_{p}$ and $\xi_{p}$ values (higher and lower than the optimum) and, as an example, in the case of the optimal tuning given by the optimisation 1-und (using (45) and (53)).

All these tests show a good match between analytical and experimental curves, proving the numerical model reliable and that it correctly describes the dynamic behaviour of the whole electromechanical system.

Once the model was validated, the results of the robustness analysis were taken into account.

The maps of the index $\varepsilon_{\text {perf }}$ were computed for both the modes and then they were compared with experimental

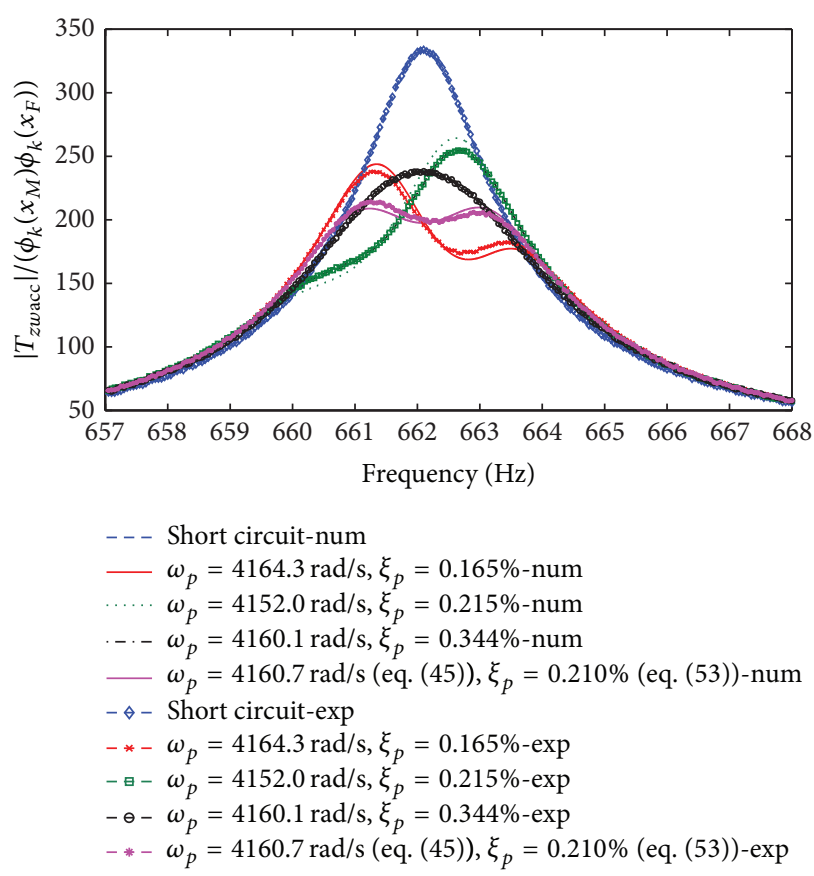

Figure 28: Numerical and experimental trend of $\left|T_{z w a c c}\right|$ (normalised over $\left.\Phi_{k}\left(x_{M}\right) \Phi_{k}\left(x_{F}\right)\right)$ for the mode at about $660 \mathrm{~Hz}$ (Table 7) and fixed values of $\omega_{p}$ and $\xi_{p}$.

results in a number of biased cases. Figure 29 shows this map in the case of the mode at about $530 \mathrm{~Hz}$. This figure also shows some bias values (points $A, B, C$, and $D$ in the figure) chosen as examples among those tested experimentally. Table 8 reports the results achieved. Furthermore, Table 9 provides the comparison between experimental and numerical results for four of the tested biased configurations for the mode at about $662 \mathrm{~Hz}$. The agreement between the experimental and the analytical results is good for both the modes.

\section{Approximated Formulation}

The previous section showed that minimisation 1 is the most robust. Yet its drawback is that it cannot have a closed analytical formulation (see Section 3.2.1) to calculate the optimal value of $\xi_{p}$ as a function of $\psi_{k}^{2} \bar{\gamma}, \xi_{k}$, and $\omega_{k}$. Therefore, 


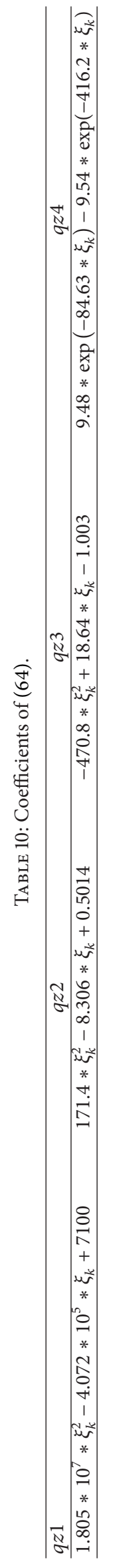


TABLE 11: Coefficients of (65).

\begin{tabular}{llll}
\hline$q m 1$ & $q m 2$ & $q m 3$ & $q m 4$ \\
\hline & & & $-1.376 * 10^{14} * \xi_{k}^{6}+4.444 * 10^{12} * \xi_{k}^{5}$ \\
$3942 * \exp \left(-404.2 * \xi_{k}\right)$ & $0.1799 * \exp \left(-161.3 * \xi_{k}\right)$ & $-0.3215 * \exp \left(-191.2 * \xi_{k}\right)$ & $-5.709 * 10^{10} * \xi_{k}^{4}$ \\
$+3137 * \exp \left(10.34 * \xi_{k}\right)$ & $+0.3206 * \exp \left(11.5 * \xi_{k}\right)$ & $-0.68 * \exp \left(8.514 * \xi_{k}\right)$ & $+3.772 * 10^{8} * \xi_{k}^{3}-1.403 * 10^{6} * \xi_{k}^{2}$ \\
& & & $+2652 \xi_{k}-0.002912$ \\
\hline
\end{tabular}

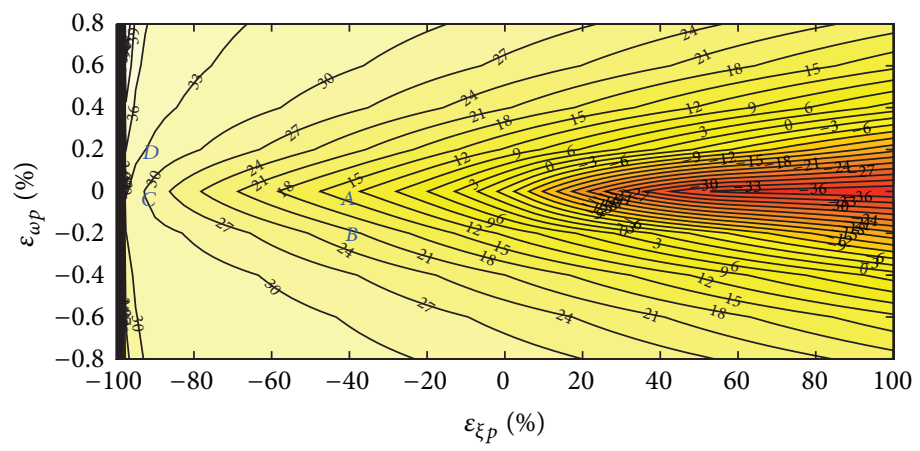

FIgURE 29: $\varepsilon_{\text {perf }}(61)$ for Mode 3 of Table 7.

this section serves to provide closed formulas achieved by means of numerical interpolation, allowing computing $\xi_{p}$ with errors under about $3 \%$ for $\psi_{k}^{2} \bar{\gamma}$ higher than $50 \mathrm{rad}^{2} / \mathrm{s}^{2}$ and under $15 \%$ for $\psi_{k}^{2} \bar{\gamma}$ lower than $50 \mathrm{rad}^{2} / \mathrm{s}^{2}$. Equation (64) gives the formulation for $\psi_{k}^{2} \bar{\gamma}$ higher than $210 \mathrm{rad}^{2} / \mathrm{s}^{2}$, while (65) for $\psi_{k}^{2} \bar{\gamma}$ lower than $210 \mathrm{rad}^{2} / \mathrm{s}^{2}$ :

$$
10^{4} \xi_{p}=q z 1 *\left(\psi_{k}^{2} \bar{\gamma}\right)^{q z 2} * \omega_{k}^{q z 3}+q z 4 .
$$

The values of $q z 1, q z 2, q z 3$, and $q z 4$ are given in Table 10:

$$
10^{4} \xi_{p}=q m 1 *\left(\psi_{k}^{2} \bar{\gamma}\right)^{q m 2} * \omega_{k}^{q m 3}+q m 4 .
$$

The values of $q m 1, q m 2, q m 3$, and $q m 4$ are given in Table 11 .

\section{Conclusion}

This paper concerns vibration attenuation by means of piezoactuators shunted to $R L$ impedances, with a major focus in performance robustness, an issue seldom accounted for in literature.

The analytical formulation that has been here proposed brings to evidence the parameter by which to drive the performance of the control method. Such a parameter depends on geometrical, mechanical, and electrical parameters of the whole system and it is not linked to the value of the eigenfrequency to control nor to the associated damping ratio. Thus, it becomes straightforward to understand how to change the actuator features, in order to increase the performance and to achieve affordable values for the electric components of the shunt impedance. Furthermore, this parameter assures the performance of the control to be asymptotic.

Furthermore, different strategies for tuning the $R L$ impedance have been compared, in terms of their performance and, overall, robustness. Such a comparison allows evidencing the most robust tuning technique; moreover, closed formulas are provided to make its practical application utmost simple. The analysis also has pointed out that it is good practice to overestimate the resistance of the impedance, to the purpose of improving attenuation robustness.

Finally experimental activity has been carried out validating the numerical results.

\section{Conflict of Interests}

The authors declare that there is no conflict of interests regarding the publication of this paper.

\section{References}

[1] N. W. Hagood and A. von Flotow, "Damping of structural vibrations with piezoelectric materials and passive electrical networks," Journal of Sound and Vibration, vol. 146, no. 2, pp. 243-268, 1991.

[2] A. Preumont, Mechatronics-Dynamics of Electromechanical and Piezoelectric Systems, Springer, Dordrecht, The Netherlands, 2006.

[3] S. Moheimani and A. Fleming, Piezoelectric Transducers for Vibration Control and Damping, Springer, London, UK, 2006.

[4] S.-Y. Wu, "Piezoelectric shunts with a parallel R-L circuit for structural damping and vibration control," in Smart Structures and Materials 1996: Passive Damping and Isolation, vol. 2720 of Proceedings of SPIE, pp. 259-269, San Diego, Calif, USA, May 1996.

[5] C. H. Park and D. J. Inman, "Uniform model for series R-L and parallel R-L shunt circuits and power consumption," in Smart Structures and Materials-Smart Structures and Integrated Systems, Proceeding of SPIE, pp. 797-804, March 1999.

[6] T. Sluka and P. Mokrý, "Feedback control of piezoelectric actuator elastic properties in a vibration isolation system," Ferroelectrics, vol. 351, no. 1, pp. 51-61, 2007. 
[7] T. Sluka, H. Kodama, E. Fukada, and P. Mokrý, "Sound shielding by a piezoelectric membrane and a negative capacitor with feedback control," IEEE Transactions on Ultrasonics, Ferroelectrics, and Frequency Control, vol. 55, no. 8, pp. 1859-1866, 2008.

[8] M. Kodejška, P. Mokrý, V. Linhart, J. Václavik, and T. Sluka, "Adaptive vibration suppression system: an iterative control law for a piezoelectric actuator shunted by a negative capacitor," IEEE Transactions on Ultrasonics, Ferroelectrics, and Frequency Control, vol. 59, no. 12, pp. 2785-2796, 2012.

[9] J. Høgsberg and S. Krenk, "Balanced calibration of resonant shunt circuits for piezoelectric vibration control," Journal of Intelligent Material Systems and Structures, vol. 23, no. 17, pp. 1937-1948, 2012.

[10] M. Redaelli, Controllo semi-passivo e semi-attivo delle vibrazioni per mezzo di trasduttori piezoelettrici laminari [Ph.D. thesis], Politecnico di Milano, Milano, Italy, 2009.

[11] A. Belloli and P. Ermanni, "Optimum placement of piezoelectric ceramic modules for vibration suppression of highly constrained structures," Smart Materials and Structures, vol. 16, no. 5, pp. 1662-1671, 2007.

[12] H. Sun, Z. Yang, K. Li et al., "Vibration suppression of a hard disk driver actuator arm using piezoelectric shunt damping with a topology-optimized PZT transducer," Smart Materials and Structures, vol. 18, no. 6, Article ID 065010, 2009.

[13] O. Thomas, J. F. Deü, and J. Ducarne, "Vibrations of an elastic structure with shunted piezoelectric patches: efficient finite element formulation and electromechanical coupling coefficients," International Journal for Numerical Methods in Engineering, vol. 80, no. 2, pp. 235-268, 2009.

[14] R. I. Bourisli and M. A. Al-Ajmi, "Optimization of smart beams for maximum modal electromechanical coupling using genetic algorithms," Journal of Intelligent Material Systems and Structures, vol. 21, no. 9, pp. 907-914, 2010.

[15] J. Ducarne, O. Thomas, and J.-F. Deü, "Placement and dimension optimization of shunted piezoelectric patches for vibration reduction," Journal of Sound and Vibration, vol. 331, no. 14, pp. 3286-3303, 2012.

[16] O. Thomas, J. Ducarne, and J.-F. Deü, "Performance of piezoelectric shunts for vibration reduction," Smart Materials and Structures, vol. 21, no. 1, Article ID 015008, 2012.

[17] J. J. Hollkamp and T. F. Starchville Jr., "Self-tuning piezoelectric vibration absorber," Journal of Intelligent Material Systems and Structures, vol. 5, no. 4, pp. 559-566, 1994.

[18] B. Zhou, F. Thouverez, and D. Lenoir, "Vibration reduction of mistuned bladed disks by passive piezoelectric shunt damping techniques," AIAA Journal, vol. 52, no. 6, pp. 1194-1206, 2014.

[19] B. Zhou, F. Thouverez, and D. Lenoir, "Essentially nonlinear piezoelectric shunt circuits applied to mistuned bladed disks," Journal of Sound and Vibration, vol. 333, no. 9, pp. 2520-2542, 2014.

[20] T. Jordan, J. Tripp, and P. Tcheng, "Electrical properties and power considerations of a piezoelectric actuator approved for public release," NASA Report, ICASE, Hampton, Va, USA, 2000.

[21] H. Hanselka, "Adaptronik und Fragen zur Systemzuverl ATP," Automatisierungstechnische Praxis, vol. 2, pp. 44-49, 2002.

[22] D. Niederberger, M. Morari, and S. Pietrzko, "Adaptive resonant shunted piezoelectric devices for vibration suppression," in Smart Structures and Materials 2003: Smart Structures and Integrated Systems, vol. 5056 of Proceedings of SPIE, pp. 213-224, March 2003.
[23] S. Behrens, A. J. Fleming, and S. O. R. Moheimani, "A broadband controller for shunt piezoelectric damping of structural vibration," Smart Materials and Structures, vol. 12, no. 1, pp. 1828, 2003.

[24] S. O. R. Moheimani, D. Halim, and A. J. Fleming, Spatial Control of Vibration, vol. 10, World Scientific Publishers, Singapore, 2003.

[25] C. Fuller, S. Elliott, and P. Nelson, Active Control of Vibration, Acadamic Press, London, UK, 1997.

[26] J. J. Dosch, D. J. Inman, and E. Garcia, "A Self-sensing piezoelectric actuator for collocated control," Journal of Intelligent Material Systems and Structures, vol. 3, no. 1, pp. 166-185, 1992.

[27] S. O. R. Moheimani, A. J. Fleming, and S. Behrens, "On the feedback structure of wideband piezoelectric shunt damping systems," Smart Materials and Structures, vol. 12, no. 1, pp. 49$56,2003$.

[28] S. Timoshenko and S. Woinowsky-Krieger, Theory of Plates and Shells, McGraw-Hill, New York, NY, USA, 1959.

[29] S. Krenk, "Frequency analysis of the tuned mass damper," Journal of Applied Mechanics, vol. 72, no. 6, pp. 936-942, 2005.

[30] J. Ormondroyd and J. P. Den Hartog, "The theory of the dynamic vibration absorber," Transactions of the American Society of Mechanical Engineers, vol. 50, pp. 9-22, 1928.

[31] E. Doebelin, Measurement Systems: Application and Design, McGraw-Hill, New York, NY, USA, 2003. 

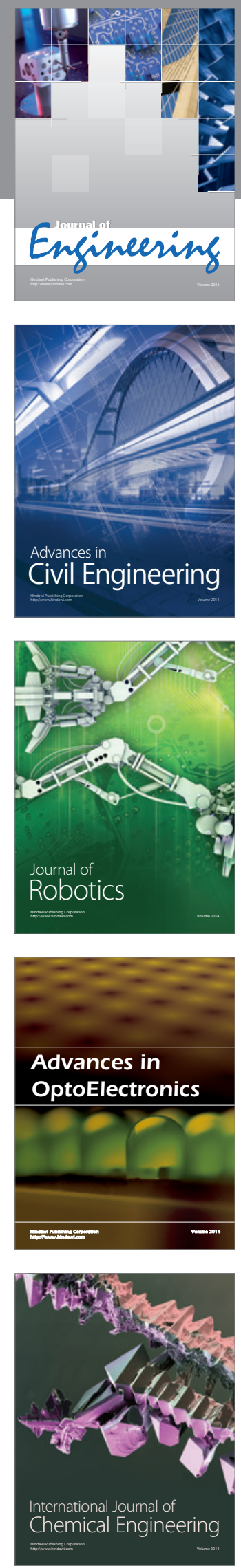

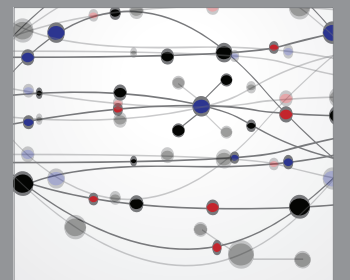

The Scientific World Journal
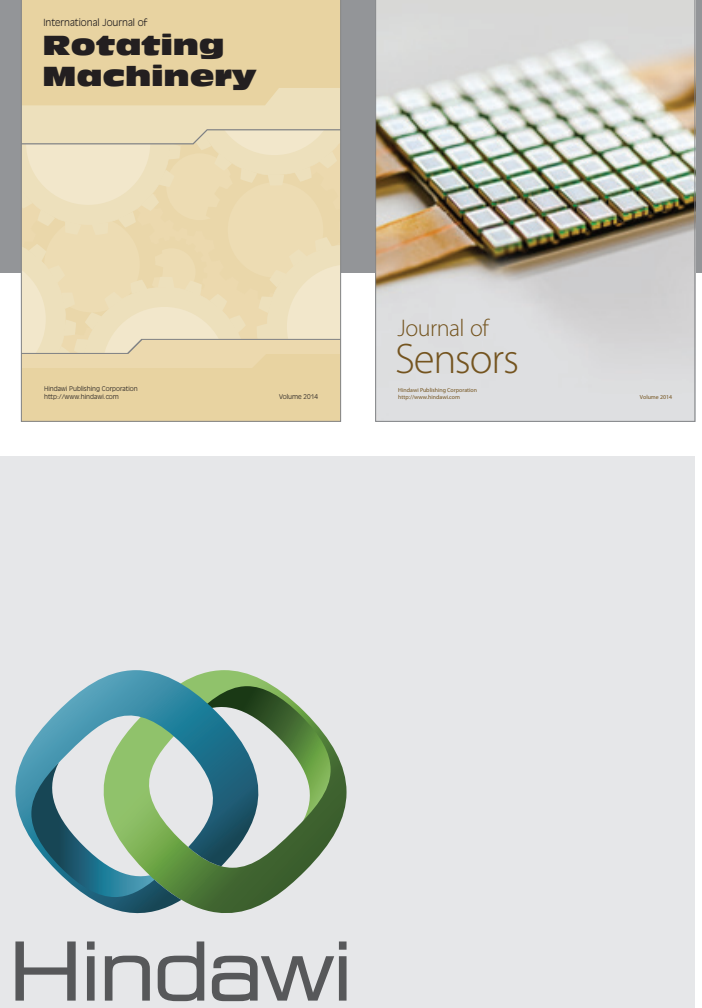

Submit your manuscripts at http://www.hindawi.com
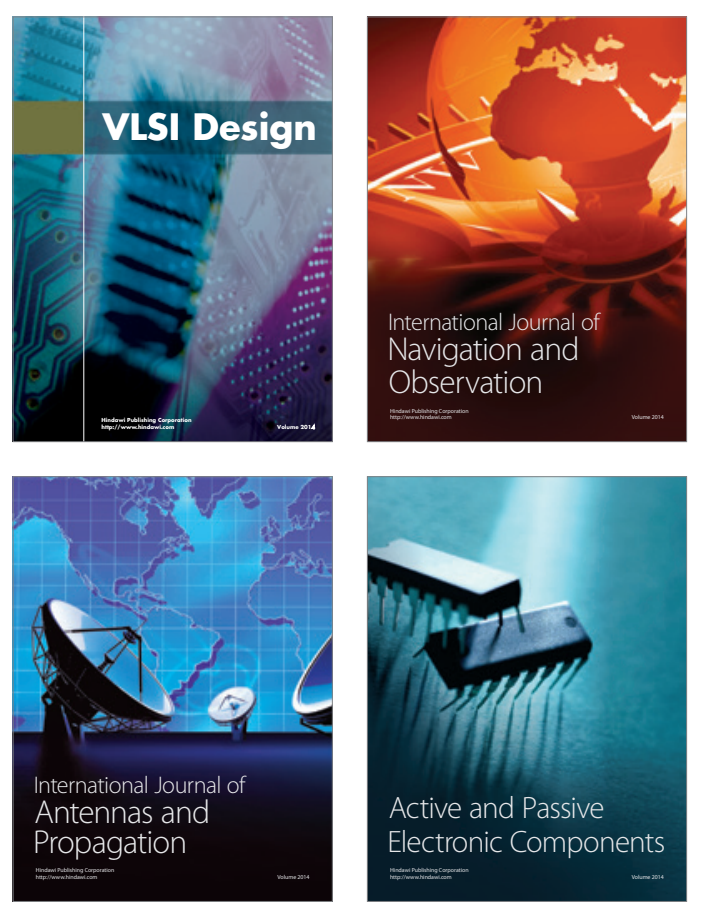
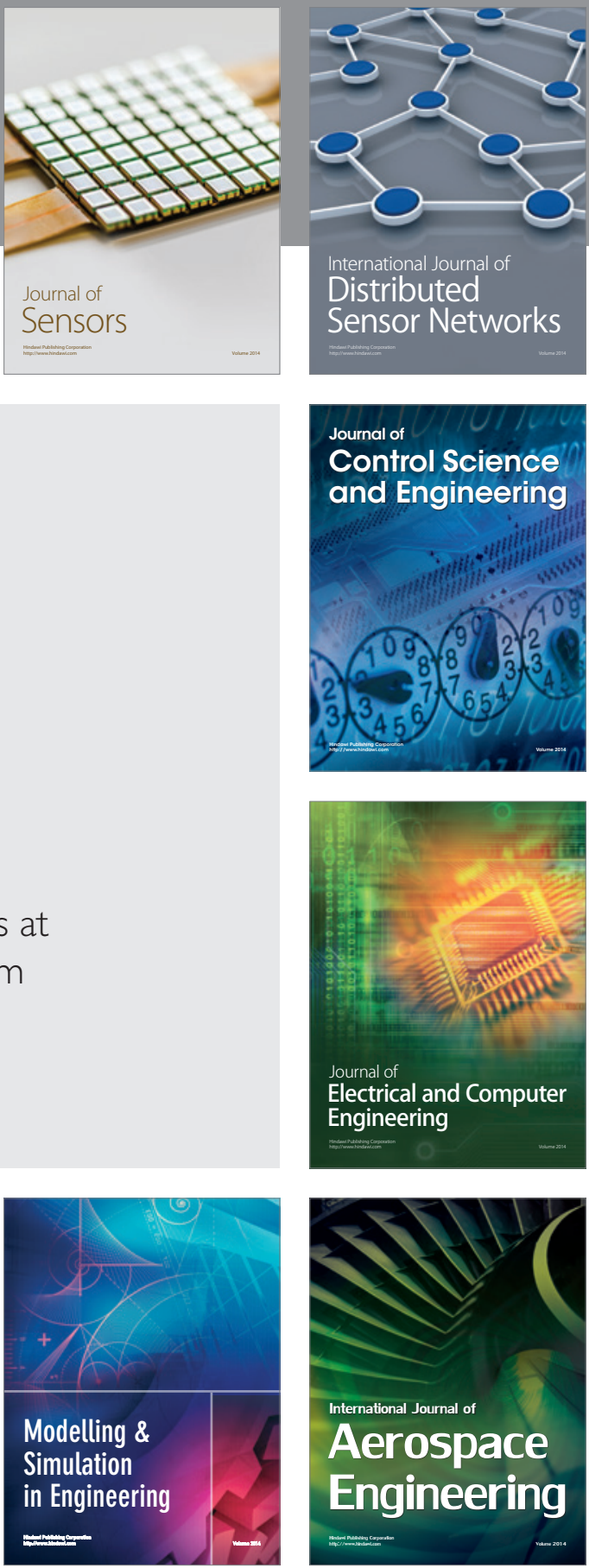

Journal of

Control Science

and Engineering
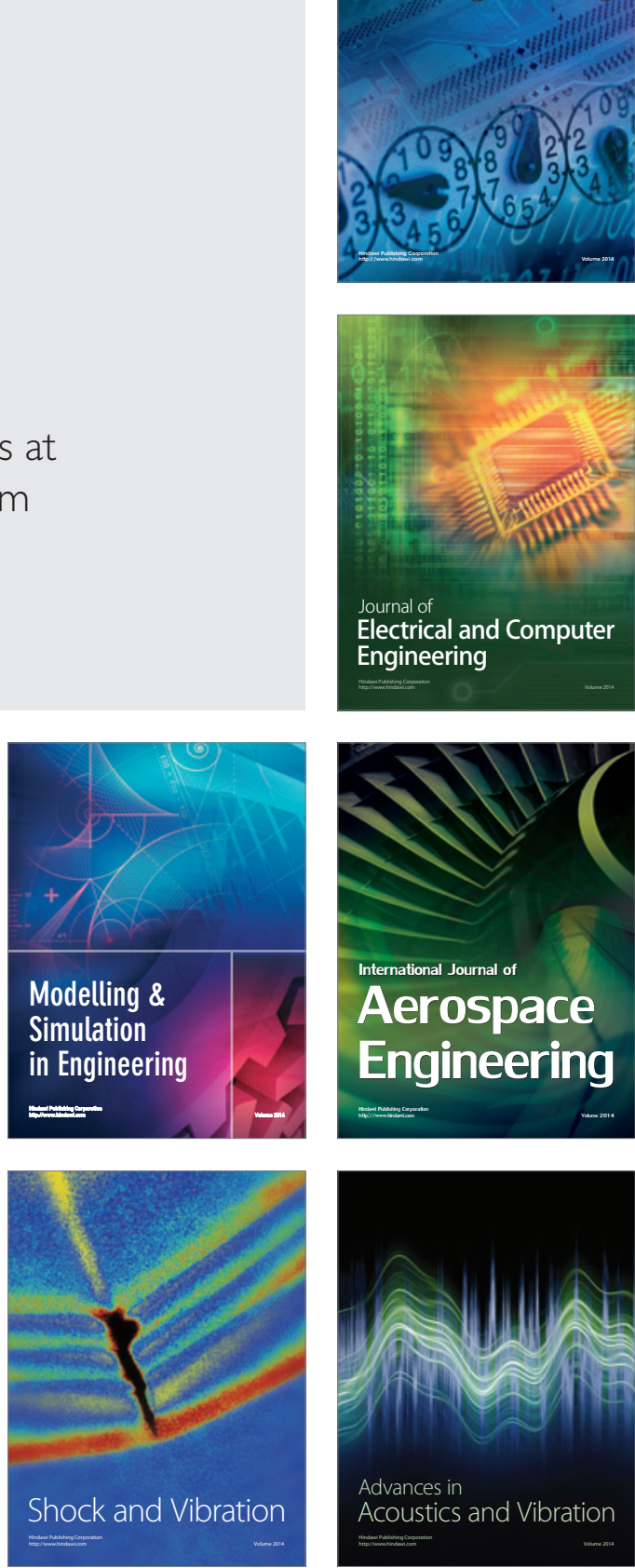